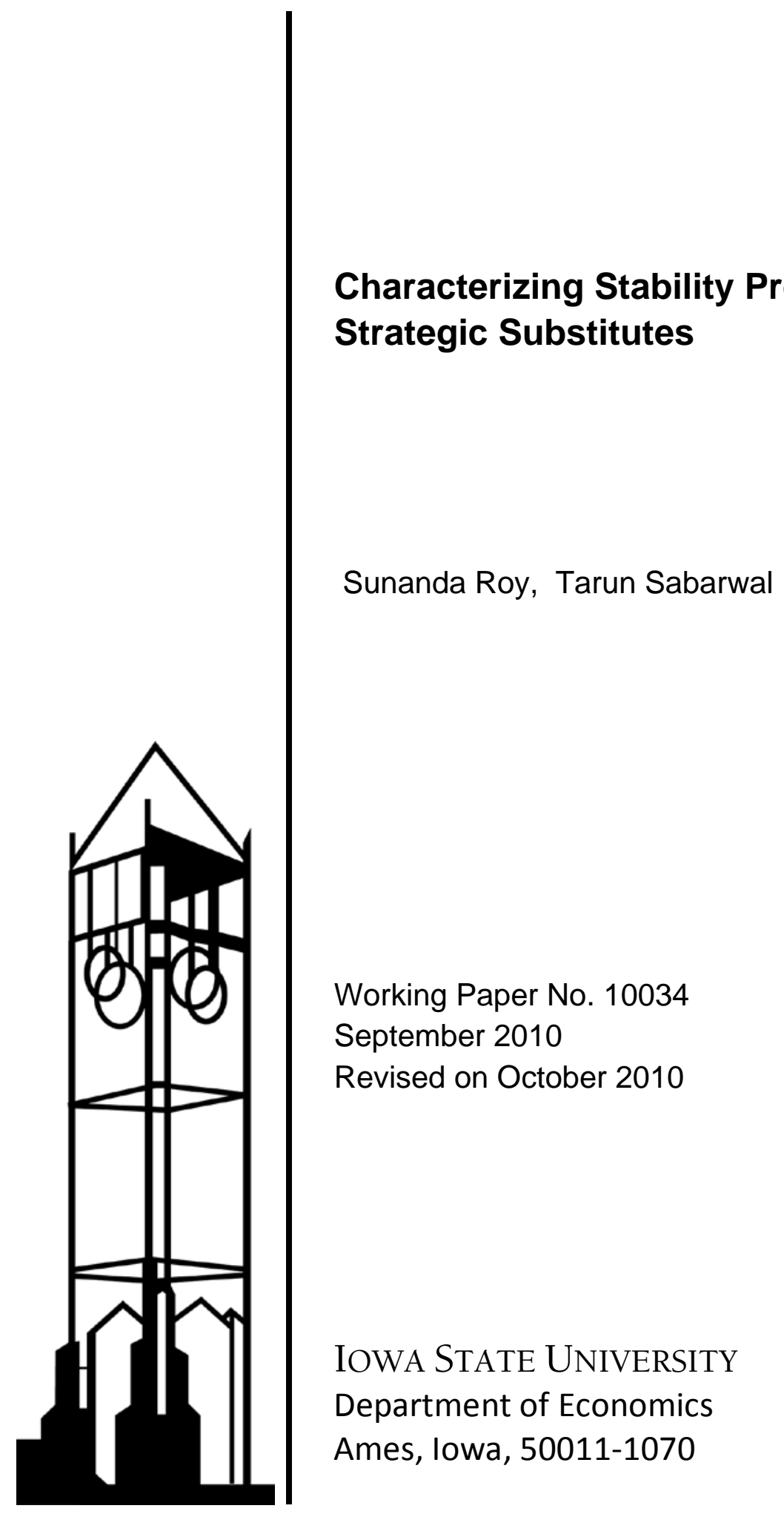

lowa State University does not discriminate on the basis of race, color, age, religion, national origin, sexual orientation, gender identity, sex, marital status, disability, or status as a U.S. veteran. Inquiries can be directed to the Director of Equal Opportunity and Diversity, 3680 Beardshear Hall, (515) 294-7612. 


\title{
Characterizing Stability Properties in Games with Strategic Substitutes*
}

\author{
Sunanda Roy \\ Department of Economics \\ Iowa State University \\ Ames IA, 50011, USA \\ sunanda@iastate.edu
}

\author{
Tarun Sabarwal \\ Department of Economics \\ University of Kansas \\ Lawrence KS, 66045, USA \\ sabarwal@ku.edu
}

\begin{abstract}
In games with strategic substitutes (GSS), convergence of the best response dynamic starting from the inf (or sup) of the strategy space is equivalent to global stability (convergence of every adaptive dynamic to the same pure strategy Nash equilibrium). Consequently, in GSS, global stability can be analyzed using a single best response dynamic. Moreover, in GSS, global stability is equivalent to dominance solvability, showing that in this class of games, two different foundations for robustness of predicted outcomes are equivalent, and both can be checked using a single best response dynamic. These equivalences are useful to study stability of equilibria in a variety of applications. Furthermore, in parameterized GSS, under natural conditions, dynamically stable equilibrium selections can be viewed in terms of monotone selections of equilibria. Several examples are provided.
\end{abstract}

JEL Numbers: C70, C72, C62

Keywords: Global stability, adaptive dynamics, strategic substitutes, dominance solvable, learning, monotone comparative statics, Correspondence principle

First Draft: July 2008

This Version: October 3, 2010

\footnotetext{
${ }^{*}$ For helpful comments on this and earlier versions of the paper, we are grateful to Rabah Amir, Federico Echenique, Kevin Reffet, Satoru Takahashi, and seminar audiences at Arizona State University, Iowa State University, the 2010 Behavioral and Quantitative Game Theory conference, the 2010 Kansas Workshop in Economic Theory, and the 2010 Midwest Economic Theory conferences.
} 


\section{Introduction}

Games with strategic substitutes (GSS) and games with strategic complements (GSC) formalize two basic economic interactions and have widespread applications. ${ }^{1}$ In GSS, bestresponse of each player is weakly decreasing in the action of each of the other players. For example, consider a Cournot oligopoly with linear demand and constant marginal costs. In GSC, best-response of each player is weakly increasing in actions of the other players. For example, consider a game of network externalities, where a given player's marginal benefit from adopting a technology is increasing as more other players adopt the same technology. ${ }^{2}$

In this paper, we focus on stability (or robustness) properties of predicted outcomes in GSS. In particular, we study stability of equilibrium, and stability of parameterized equilibrium selections.

With regard to stability of equilibrium, we first show that in GSS, convergence of the best response dynamic starting from the inf (or sup) of the strategy space is equivalent to convergence of every adaptive dynamic ${ }^{3}$ to the same (pure strategy) Nash equilibrium. In other words, in GSS, convergence of the best response dynamic from inf (or sup) of the strategy space is equivalent to global stability, where global stability is defined as convergence of every adaptive dynamic to the same (pure strategy) Nash equilibrium.

This result provides a new perspective on global stability. In GSS, knowledge of convergence of a single best response dynamic yields convergence of all adaptive dynamics to the same outcome. Consequently, whether players actually play best response dynamics or not, convergence of a single best response dynamic is sufficient to conclude convergence under all adaptive behavior. This provides an alternative to the traditional eigen-value approach applied to each dynamic in a class of dynamics, as in, for example, Al-Nowaihi and Levine

\footnotetext{
${ }^{1}$ Such games are defined in Bulow, Geanakoplos, and Klemperer (1985), and as they show, models of strategic investment, entry deterrence, technological innovation, dumping in international trade, natural resource extraction, business portfolio selection, and others can be viewed in a more unifying framework according as the variables under consideration are strategic complements or strategic substitutes. Earlier developments are provided in Topkis (1978) and Topkis (1979).

Versions of such games arise in diverse economic environments, including competitive strategy, public goods, industrial organization, natural resource utilization, manufacturing analysis, team management, tournaments, resource allocation, business portfolio development, principal-agent modeling, multi-lateral contracting, auctions, technological innovation, behavioral economics, and others.

2 There is a long literature developing the theory of GSC. Some of this work can be seen in Topkis (1978), Topkis (1979), Lippman, Mamer, and McCardle (1987), Sobel (1988), Milgrom and Roberts (1990), Vives (1990), Zhou (1994), Milgrom and Shannon (1994), Milgrom and Roberts (1994), Shannon (1995), VillasBoas (1997), Edlin and Shannon (1998), Echenique (2002), Echenique and Sabarwal (2003), Quah (2007), and Quah and Strulovici (2009), among others. Extensive bibliographies are available in Topkis (1998), in Vives (1999), and in Vives (2005).

For some recent research on GSS, confer Amir (1996), Villas-Boas (1997), Amir and Lambson (2000), Schipper (2003), Zimper (2007), Roy and Sabarwal (2008), Acemoglu and Jensen (2009), Acemoglu and Jensen (2010), Roy and Sabarwal (2010), and Jensen (2010), among others.

${ }^{3}$ Recall that adaptive dynamics allow for strategic behavior and learning processes with very few restrictions. Intuitively, the only requirement in an adaptive dynamic is that eventually, future play should be an undominated response to the order interval determined by past play, (or at least in the order interval determined by such undominated responses.)
} 
(1985) and Okuguchi and Yamazaki (2008).

Next, we provide a connection to existing results on dominance solvability in GSS. Zimper (2007) shows that in GSS, there exist smallest and largest serially undominated strategies, and a GSS is dominance solvable iff the second iterate of the (joint) best response function has a unique fixed point. We use a more general model than Zimper (2007), and show that his results go through for our generalizations. In particular, in GSS, convergence of the best response dynamic from inf (or sup) of the strategy space is equivalent to dominance solvability.

Connecting these two results, we conclude that in GSS, global stability is equivalent to dominance solvability. This brings together two different foundations for robustness of predicted outcomes in games. Dominance solvability (and rationalizability) assume fully informed players, using infinite iterations of rationalizing about potential (future) responses by competitors to predict a solution to a one-shot game. Global stability uses dynamic learning and strategic processes in a series of game-play over time, using past play by typically myopic players to determine present moves, and relying on limits of such learning and strategic behavior to predict an outcome robust to the dynamics. In GSS, both approaches are equivalent, and moreover, both global stability and dominance solvability can be checked using a single best response dynamic. ${ }^{4}$

Recall that Moulin (1984) has shown that in all strategic games, dominance solvability implies Cournot stability (convergence of all best-response dynamics), and that Zimper (2007)'s results imply that in GSS, Cournot stability is equivalent to dominance solvability. Our results extend this to show that in GSS, global stability (convergence of every adaptive dynamic, not just best response dynamics) is equivalent to dominance solvability.

In addition to showing the theoretical equivalence of these approaches, we show that these results can be used profitably in a variety of applications in diverse areas. We present applications to games with linear best responses (such as simple Cournot oligopoly, commonpool resource games, differentiated goods Cournot oligopoly with finitely many firms, and private provision of public goods with finitely many consumers), games with non-linear best responses (such as general, symmetric, homogeneous goods Cournot oligopoly, tournaments, general two-player GSS, and general two-player GSC), and discrete games (such as DoveHawk-Chicken-type games). In each case, knowledge of a particular aspect of the game allows for powerful cross-derivation of results.

With regard to stability of equilibrium selections, we show that in parameterized GSS, monotone equilibrium selections are dynamically stable, in the following sense.

We show that in parameterized GSS, continuous and strictly increasing equilibrium selections select strongly stable equilibria, under natural conditions. (Intuitively, an equilibrium is strongly stable if it has a neighborhood such that every adaptive dynamic starting in this neighborhood converges to it.) In particular, small changes in the parameter are dynamically stable, because at a new parameter value, every adaptive dynamic starting from the

\footnotetext{
${ }^{4}$ Using Milgrom and Roberts (1990), it is easy to deduce that global stability is equivalent to dominance solvability in GSC as well. What is not true in GSC is the equivalence of convergence of the best response dynamic from inf (or sup) of strategy space to global stability, or to dominance solvability.
} 
old equilibrium converges to the newly selected equilibrium.

Similarly, we show that continuous and nowhere weakly increasing equilibrium selections select equilibria that are not even weakly stable, under similar conditions. (Intuitively, an equilibrium is weakly stable if it has a neighborhood such that some adaptive dynamic starting in this neighborhood converges to it.) In particular, changes in the parameter are dynamically unstable, because at a new parameter value, no adaptive dynamic starting from the old equilibrium converges to the newly selected equilibrium.

Thus, when considering dynamically stable equilibria (as proposed by Samuelson's Correspondence principle), we may expect monotone selections of equilibria to arise naturally in GSS. Echenique (2002) provides a similar result for parameterized GSC.

In addition to these results, we clarify two aspects of the theory of GSS.

First, we show that a GSS may not necessarily have a (pure strategy) Nash equilibrium. We present a three-player, two-action, Dove-Hawk-Chicken-type game with no pure-strategy Nash equilibrium. This shows that a GSS cannot always be viewed as an aggregative game, or as a GSC, because such games always have a PSNE. In particular, the standard technique of reversing the order on the strategy space of one player in a GSS to yield a GSC does not extend to more than two players.

Second, fixed points of the second iterate of the joint best-responses play a significant role in the analysis of GSS. These may be motivated in terms of simply rationalizable strategies. Intuitively, a simply rationalizable strategy profile is one that can be simultaneously rationalized by no more than two iterations of behavioral conjectures. They may be viewed as outcomes of low-level rationalization, exhibiting a type of bounded rationality. ${ }^{5}$ Simply rationalizable profiles provide a behavioral interpretation for such fixed points. In this terminology, in GSS, simple rationalization always predicts an outcome, even if a GSS has no PSNE. Moreover, if simple rationalization predicts a unique outcome, then higher-level rationalization has no additional benefit.

The paper proceeds as follows. Section 2 presents the results on global stability, and their connection to dominance solvability. Section 3 provides several applications. Section 4 presents the results on stability of monotone equilibrium selections.

\section{Stability of Equilibrium}

Let $I$ be a non-empty set of players, and for each player $i$, a strategy space that is a partially ordered set $\left(X^{i}, \preceq^{i}\right)$, and a real-valued payoff function, denoted $f^{i}\left(x_{i}, x_{-i}\right)$. As usual, the domain of each $f^{i}$ is the product of the strategy spaces, $(X, \preceq)$ endowed with the product order. ${ }^{6}$ The strategic game $\Gamma=\left\{I,\left(X^{i}, \preceq^{i}, f^{i}\right)_{i \in I}\right\}$ is a game with strategic substitutes

\footnotetext{
${ }^{5}$ Nash equilibrium profiles are simply rationalizable, and simply rationalizable profiles are rationalizable; both inclusions may be strict.

${ }^{6}$ The topology on $X^{i}$ is the standard order interval topology, and the topology on $X$ is the product topology. For notational convenience, we shall sometimes drop the index $i$ from the notation for the partial order.
} 
$(\boldsymbol{G S S})$, if for every player $i$

1. $X^{i}$ is a complete lattice,

2. For every $x_{-i}, f^{i}$ is order upper semi-continuous in $x_{i}$, and for every $x_{i}, f^{i}$ is order continuous in $x_{-i}$,

3. For every fixed $x_{-i}, f^{i}$ is quasi-supermodular in $x_{i},{ }^{7}$ and

4. $f^{i}$ satisfies the decreasing single-crossing property in $\left(x_{i} ; x_{-i}\right){ }^{8}$

As compared to Milgrom and Roberts (1990), we have replaced supermodular with its ordinal generalization, quasi-supermodular, and replaced increasing differences with the decreasing single-crossing property, an ordinal generalization of decreasing differences. The decreasing single-crossing property captures the idea of strategic substitutes, just as the single-crossing property formalizes the idea of strategic complements.

For each player $i$, the best response of player $i$ is denoted $g^{i}\left(x_{-i}\right)$. As is well-known (see, for example, Milgrom and Shannon (1994)), for each player $i$, the best response of player $i, g^{i}\left(x_{-i}\right)$, is a non-empty, complete lattice. Let $\bar{g}^{i}\left(x_{-i}\right)=\sup g^{i}\left(x_{-i}\right)$ and $\underline{g}^{i}\left(x_{-i}\right)=$ $\inf g^{i}\left(x_{-i}\right)$ be the extremal best responses. As is well-known (see, for example, Topkis (1998)), for each player $i$, and for each profile of other player strategies $x_{-i}, g^{i}\left(x_{-i}\right)$ is nonincreasing in $x_{-i},{ }^{9}$ and therefore, for each player $i$, both $\bar{g}^{i}\left(x_{-i}\right)$ and $g^{i}\left(x_{-i}\right)$ are nonincreasing functions. ${ }^{10}$ Let $g: X \rightarrow X, g(x)=\left(g^{i}\left(x_{-i}\right)\right)_{i \in I}$, denote the joint best-response correspondence. Then the correspondence $g$ is nonincreasing, ${ }^{11}$ and the functions $\underline{g}(x)=\inf g(x)$ and $\bar{g}(x)=$ $\sup g(x)$ are nonincreasing.

As usual, a (pure strategy) Nash equilibrium of the game is a profile of player actions $x$ such that $x \in g(x)$. The equilibrium set of the game is given by $\mathcal{E}=\{x \in X \mid x \in g(x)\}$. It is possible that a GSS has no (pure strategy) Nash equilibrium. Consider the following example.

Example 1 (Dove-Hawk-Chicken). Consider a three-player version of a Dove-HawkChicken-type game, as follows. Suppose there are three players, and each player has a choice between two actions: D (Dove) is the "lower" action (more dovish, more accommodating, less aggressive action), and $\mathrm{H}$ (Hawk) is the "higher" action (more hawkish, less accommodating, more aggressive action). Payoffs are given in figure 1.

${ }^{7}$ As in Milgrom and Shannon (1994), a function $f: X \rightarrow \mathbb{R}$ (where $X$ is a lattice) is quasi-supermodular if (1) $f(x) \geq f(x \wedge y) \Longrightarrow f(x \vee y) \geq f(y)$, and $(2) f(x)>f(x \wedge y) \Longrightarrow f(x \vee y)>f(y)$.

${ }^{8}$ A function $f: X \times T \rightarrow \mathbb{R}$ (where $X$ is a lattice and $T$ is a partially ordered set) satisfies decreasing single-crossing property in $(x ; t)$ if for every $x^{\prime} \succ x^{\prime \prime}$ and $t^{\prime} \succ t^{\prime \prime},(1) f\left(x^{\prime}, t^{\prime \prime}\right) \leq f\left(x^{\prime \prime}, t^{\prime \prime}\right) \Longrightarrow f\left(x^{\prime}, t^{\prime}\right) \leq$ $f\left(x^{\prime \prime}, t^{\prime}\right)$, and $(2) f\left(x^{\prime}, t^{\prime \prime}\right)<f\left(x^{\prime \prime}, t^{\prime \prime}\right) \Longrightarrow f\left(x^{\prime}, t^{\prime}\right)<f\left(x^{\prime \prime}, t^{\prime}\right)$. This property is discussed in some detail in Roy and Sabarwal (2010). Amir (1996) terms this property the dual single-crossing property.

${ }^{9}$ For every $x_{-i}$ and $x_{-i}^{\prime}$, if $x_{-i} \preceq x_{-i}^{\prime}$ then $g^{i}\left(x_{-i}^{\prime}\right) \sqsubseteq^{i} g^{i}\left(x_{-i}\right)$, where the order on nonempty subsets of $X^{i}$ is the standard (induced) set order used in the literature. That is, for non-empty subsets $A, B$ of $X^{i}$, $A \sqsubseteq^{i} B$ if for every $a \in A$, and for every $b \in B, a \wedge b \in A$, and $a \vee b \in B$, where the operations $\wedge, \vee$ are with respect to $\preceq^{i}$.

${ }^{10}$ For every $x_{-i}$ and $x_{-i}^{\prime}$, if $x_{-i} \preceq x_{-i}^{\prime}$ then $\bar{g}^{i}\left(x_{-i}^{\prime}\right) \preceq \bar{g}^{i}\left(x_{-i}\right)$ and $\underline{g}^{i}\left(x_{-i}^{\prime}\right) \preceq \underline{g}^{i}\left(x_{-i}\right)$.

${ }^{11}$ In the standard induced set order, as in Topkis (1998). 


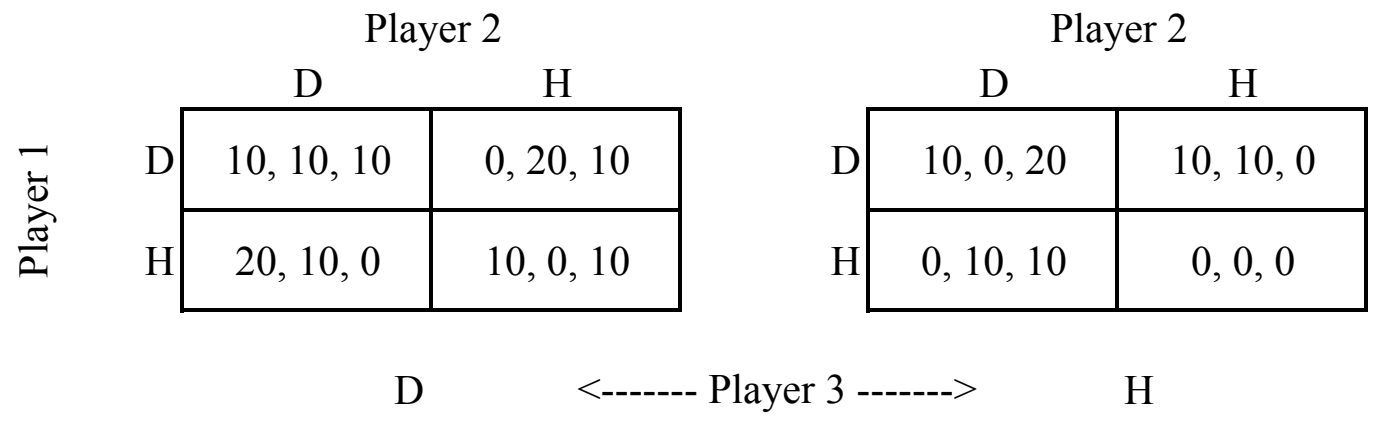

Figure 1: A GSS with no (pure strategy) Nash equilibrium

Notice that each player wishes to be (weakly) less aggressive, the more aggressive are his competitors. This game is an extension of a two-player, Dove-Hawk-Chicken game. Indeed, if we fix the action of any one player, then the remaining two-player game is a version of a standard Dove-Hawk-Chicken game that has a unique Nash equilibrium - one player plays $\mathrm{D}$ and the other plays $\mathrm{H}$. With three players, it is easy to check that this game has no (pure strategy) Nash equilibrium.

Example 1 clarifies another aspect of the theory of GSS. A GSS cannot always be viewed as an aggregative game, ${ }^{12}$ or as a GSC, because both types of games always have a purestrategy Nash equilibrium. In particular, the standard technique of reversing the order on the strategy space of one player in a GSS to yield a GSC does not extend to more than two players.

Let's now develop bounds on undominated strategies. As usual, ${ }^{13}$ a pure strategy $x_{i} \in X^{i}$ is strongly dominated, if there exists $\hat{x}_{i} \in X^{i}$ such that for every $x_{-i}, f^{i}\left(x_{i}, x_{-i}\right)<$ $f^{i}\left(\hat{x}_{i}, x_{-i}\right)$. For a given set of strategy profiles $\hat{X} \subset X$, player $i$ 's undominated responses to $\hat{X}$ is the set

$$
U_{i}(\hat{X})=\left\{x_{i} \in X^{i} \mid \forall x_{i}^{\prime} \in X^{i}, \exists \hat{x} \in \hat{X}, f^{i}\left(x_{i}, \hat{x}_{-i}\right) \geq f^{i}\left(x_{i}^{\prime}, \hat{x}_{-i}\right)\right\} .
$$

Let $U(\hat{X})=\left(U_{i}(\hat{X})_{i \in I}\right)$ denote the collection of undominated responses to $\hat{X}$, one for each player, and let $\bar{U}(\hat{X})=[\inf U(\hat{X})$, sup $U(\hat{X})]$ be the smallest order interval containing $U(\hat{X})$. Higher-order undominated strategies are defined iteratively, as follows: $U^{0}(\hat{X})=\hat{X}$, and for $k \geq 1, U^{k}(\hat{X})=U^{k-1}(\hat{X})$. Building on techniques from Milgrom and Roberts (1990), the following lemma highlights the structure of the smallest order interval containing undominated strategies.

Lemma 1. For every $a \preceq b$ in $X, \bar{U}[a, b]=[\underline{g}(b), \bar{g}(a)]$.

Proof. Let's first see that $U[a, b] \subset[\underline{g}(b), \bar{g}(a)]$. Consider the contrapositive. Suppose $y \notin[\underline{g}(b), \bar{g}(a)]$. Then either, $y \npreceq \bar{g}(a)$ or $y \nsucceq \underline{g}(b)$. Suppose $y \npreceq \bar{g}(a)$. In particular, consider player $i$ such that $y_{i} \npreceq \bar{g}^{i}\left(a_{-i}\right)$. Then $y_{i} \wedge \bar{g}^{i}\left(\bar{a}_{-i}\right)$ dominates $y_{i}$, as follows. Indeed, for every

\footnotetext{
${ }^{12}$ Confer Dubey, Haimanko, and Zapechelnyuk (2006), or Jensen (2010).

${ }^{13}$ Following Milgrom and Roberts (1990).
} 
$x \in[a, b]$,

$$
\begin{aligned}
f^{i}\left(y_{i} \vee\right. & \left.\bar{g}^{i}\left(a_{-i}\right), a_{-i}\right)-f^{i}\left(\bar{g}^{i}\left(a_{-i}\right), a_{-i}\right)<0 \\
& \Longrightarrow \quad f^{i}\left(y_{i}, a_{-i}\right)-f^{i}\left(y_{i} \wedge \bar{g}^{i}\left(a_{-i}\right), a_{-i}\right)<0 \\
& \Longrightarrow \quad f^{i}\left(y_{i}, x_{-i}\right)-f^{i}\left(y_{i} \wedge \bar{g}^{i}\left(a_{-i}\right), x_{-i}\right)<0
\end{aligned}
$$

where the first inequality follows from the definition of $\bar{g}^{i}(a)$, the first implication follows from quasi-supermodularity, and the second implication follows from decreasing single-crossing property. The case $y \nsucceq \underline{g}(b)$ follows similarly. Thus, $y \notin U[a, b]$, whence $U[a, b] \subset[g(b), \bar{g}(a)]$. Therefore, $\bar{U}[a, b] \subset[\underline{g}(\bar{b}), \bar{g}(a)]$. Moreover, as g is a best response, $\underline{g}(b)$ and $\bar{g}(a)$ are in $U[a, b]$, whence $[\underline{g}(b), \bar{g}(a)] \subset \bar{U}[a, b]$.

Lemma 1 shows that adjusting for strategic substitutes reverses the relationship that holds for strategic complements. ${ }^{14}$ This provides an important insight into the appropriate design of best-response dynamics that bound higher-order undominated strategies, as follows. Suppose $y^{0}=\inf X$ and $z^{0}=\sup X$. Then $U^{0}(X) \subset\left[y^{0}, z^{0}\right]=X$. Lemma 1 shows that $U^{1}(X) \subset\left[\underline{g}\left(z^{0}\right), \bar{g}\left(y^{0}\right)\right]$, and $\bar{U}^{1}(X)=\left[\underline{g}\left(z^{0}\right), \bar{g}\left(y^{0}\right)\right]$. Thus, at the first iteration, it is useful to transform $y^{0}=\inf X$ to $y^{1}=\bar{g}\left(y^{0}\right)$ and $z^{0}=\sup X$ to $z^{1}=\underline{g}\left(z^{0}\right)$. Similarly, another iteration of the lemma yields

$$
U^{2}(X)=U(U(X)) \subset U\left(\left[z^{1}, y^{1}\right]\right) \subset \bar{U}\left(\left[z^{1}, y^{1}\right]\right)=\left[\underline{g}\left(y^{1}\right), \bar{g}\left(z^{1}\right)\right] .
$$

Thus, at the second iteration, it is useful to transform $y^{1}$ to $y^{2}=\underline{g}\left(y^{1}\right)$, and $z^{1}$ to $z^{2}=\bar{g}\left(z^{1}\right)$. The process continues inductively. This is the construction followed here, and it turns out be very useful to analyze GSS. ${ }^{15}$

The (simultaneous) best response dynamic starting at inf $X$ is the sequence $\left(y^{k}\right)_{k=0}^{\infty}$ given by $y^{0}=\inf X$, and for $k \geq 1, y^{k}=\underline{g}\left(y^{k-1}\right)$ if $k$ is even, and $\bar{g}\left(y^{k-1}\right)$ if $k$ is odd. Notice that when $g$ is a best-response function, $\left(y^{k}\right)$ is the standard simultaneous best-response dynamic starting at $\inf X$. When $g$ is a correspondence, lemma 1 shows that this is the appropriate definition.

Similarly, the (simultaneous) best response dynamic starting at $\sup X$ is the sequence $\left(z^{k}\right)_{k=0}^{\infty}$ given by $z^{0}=\sup X$, and for $k \geq 1, z^{k}=\bar{g}\left(z^{k-1}\right)$ if $k$ is even, and $\underline{g}\left(z^{k-1}\right)$ if $k$ is odd. Again, when $g$ is a best-response function, $\left(z^{k}\right)$ is the standard simultaneous best-response dynamic starting at $\sup X$.

Mixtures of the sequences $\left(y^{k}\right)$ and $\left(z^{k}\right)$ help to provide bounds on adaptive dynamics, as follows. The lower mixture of $\left(\left(y^{k}\right) ;\left(z^{k}\right)\right)$ is the sequence $\left(\underline{x}^{k}\right)_{k=0}^{\infty}$ given by $\underline{x}^{k}=y^{k}$, if

\footnotetext{
${ }^{14}$ In a GSC, the corresponding result from Milgrom and Roberts (1990) is as follows: for every $a, b \in X$ such that $a \preceq b, \bar{U}[a, b]=[\underline{g}(a), \bar{g}(b)]$.

${ }^{15}$ Compare this with the construction in Milgrom and Roberts (1990), where $y^{0}=\inf X$, and $z^{0}=\sup X$, and for $k \geq 1, y^{k}=\underline{g}\left(y^{k-1}\right)$, and $z^{k}=\bar{g}\left(z^{k-1}\right)$. For GSC, this construction yields the following useful facts: $\left(y^{k}\right)$ is monotone nondecreasing, $\left(z^{k}\right)$ is monotone nonincreasing, and these sequences are comparable all along; that is, for every $k, y^{k} \preceq z^{k}$. For GSS, this construction does not get us very far. In this case, $y^{0} \preceq z^{0}$, and then $y^{0} \preceq y^{1}$, but then $y^{2} \preceq y^{1}$, and then $y^{2} \preceq y^{3}$, and $y^{4} \preceq y^{3}$, and so on. Thus, a monotonic relationship in the progression of elements in either sequence does not emerge. Moreover, if we consider the second-iterate of this construction, then it is true that $y^{0} \preceq y^{2}$, and inductively, for every $k, y^{2 k} \preceq y^{2 k+2}$, and therefore, the sequence $\left(y^{2 k}\right)$ is nondecreasing, and similarly, $\left(z^{2 k}\right)$ is nonincreasing. But with nonincreasing $g$, it does not follow that in general, $\underline{g} \circ \underline{g}\left(y^{0}\right) \preceq \bar{g} \circ \bar{g}\left(z^{0}\right)$, and therefore, a clear comparison across the sequences $\left(y^{k}\right)$ and $\left(z^{k}\right)$ does not emerge.
} 
$k$ is even, and $\underline{x}^{k}=z^{k}$, if $k$ is odd, and the upper mixture of $\left(\left(y^{k}\right) ;\left(z^{k}\right)\right)$ is the sequence $\left(\bar{x}^{k}\right)_{k=0}^{\infty}$ given by $\bar{x}^{k}=z^{k}$, if $k$ is even, and $\bar{x}^{k}=y^{k}$, if $k$ is odd. ${ }^{16}$ These mixed sequences have some useful properties, as shown in the following lemma.

Lemma 2. Let $\left(y^{k}\right)$ and $\left(z^{k}\right)$ be the best response dynamics starting at inf $X$ and $\sup X$, respectively, and $\left(\underline{x}^{k}\right)$ and $\left(\bar{x}^{k}\right)$ be their lower and upper mixtures, respectively.

1. The sequence $\left(\underline{x}^{k}\right)$ is nondecreasing and there is $\underline{x}$ such that $\underline{x}=\lim _{k} \underline{x}^{k}$.

2. The sequence $\left(\bar{x}^{k}\right)$ is nonincreasing and there is $\bar{x}$ such that $\bar{x}=\lim _{k} \bar{x}^{k}$.

3. For every $k, \underline{x}^{k} \preceq \bar{x}^{k}$.

Proof. For statements (1) and (2), notice that if $\underline{x}^{k} \preceq \underline{x}^{k+1}$, then $\bar{x}^{k+1}=\bar{g}\left(\underline{x}^{k}\right) \succeq \bar{g}\left(\underline{x}^{k+1}\right)=$ $\bar{x}^{k+2}$, and similarly, if $\bar{x}^{k} \succeq \bar{x}^{k+1}$, then $\underline{x}^{k+1} \preceq \underline{x}^{k+2}$. Thus, the sequence $\left(\underline{x}^{k}\right)$ is nondecreasing, and the sequence $\left(\bar{x}^{k}\right)$ is nonincreasing, if $\underline{x}^{0} \preceq \underline{x}^{1}$, and $\bar{x}^{0} \succeq \bar{x}^{1}$. But this is true, because $\underline{x}^{0}=\inf X$, and $\bar{x}^{0}=\sup X$. As $X$ is complete, each of these sequences converges in $X$.

Statement (3) holds trivially for $k=0$. Suppose $\underline{x}^{k} \preceq \bar{x}^{k}$. Then $\underline{x}^{k+1}=\underline{g}\left(\bar{x}^{k}\right) \preceq \underline{g}\left(\underline{x}^{k}\right) \preceq$ $\bar{g}\left(\underline{x}^{k}\right)=\bar{x}^{k+1}$.

To define an adaptive dynamic, we follow Milgrom and Roberts (1990). A process $(x(k))_{k \in \hat{K}}$ in $\Gamma$ is an adaptive dynamic in $\Gamma$ if for every $K$, there is $K^{\prime}$ such that for every $k \geq K^{\prime}, x(k) \in \bar{U}[\inf P(K, k)$, sup $P(K, k)]$. Here, $P(K, k)$ is the set of past play from $K$ up to (but not including) $k$; that is, $P(K, k)=\{x(\xi) \mid K \leq \xi<k\}$.

Notice that the definition of an adaptive dynamic puts very few restrictions on strategic or learning behavior. All it requires is that eventually, future play should be an undominated response to the order interval determined by past play, or at least be in the order interval determined by such undominated responses. In particular, adaptive dynamics include simultaneous Cournot dynamics, sequential Cournot dynamics, fictitious play, tatonnement-type price adjustment dynamics, and they allow for various learning behavior, "mistakes," and other "out-of equilibrium" dynamics. The following result provides bounds on eventual behavior of adaptive dynamics in GSS.

Lemma 3. Let $\left(\underline{x}^{k}\right)$ and $\left(\bar{x}^{k}\right)$ be the lower and upper mixtures of $\left(\left(y^{k}\right) ;\left(z^{k}\right)\right)$, respectively, and let $\underline{x}$ and $\bar{x}$ be their respective limits. For every adaptive dynamic $(x(k))$ in $\Gamma$,

1. For every $N$, there is $K_{N}$ such that for all $k \geq K_{N}, x(k) \in\left[\underline{x}^{N}, \bar{x}^{N}\right]$.

2. $\underline{x} \preceq \liminf x(k) \preceq \limsup x(k) \preceq \bar{x}$.

Moreover, if each player has a finite strategy space, then there is $K^{*}$ such that for every $k \geq K^{*}, \underline{x} \preceq x(k) \preceq \bar{x}$.

\footnotetext{
${ }^{16}$ In other words, $\underline{x}^{0}=\inf X, \bar{x}^{0}=\sup X$, and for $k \geq 1, \underline{x}^{k}=\underline{g}\left(\bar{x}^{k-1}\right)$, and $\bar{x}^{k}=\bar{g}\left(\underline{x}^{k-1}\right)$.
} 
Proof. Statement (1) holds trivially for $N=0$. Suppose there is $K_{N-1}$ such that for all $k \geq K_{N-1}, x(k) \in\left[\underline{x}^{N-1}, \bar{x}^{N-1}\right]$. Then for all $k \geq K_{N-1},\left[\inf P\left(K_{N-1}, k\right), \sup P\left(K_{N-1}, k\right)\right] \subset$ $\left[\underline{x}^{N-1}, \bar{x}^{N-1}\right]$. Now, by definition of an adaptive dynamic, let $K_{N}$ be such that for all $k \geq K_{N}$, $x(k) \in \bar{U}\left[\inf P\left(K_{N-1}, k\right), \sup P\left(K_{N-1}, k\right)\right]$, and consequently, for all $k \geq K_{N}$,

$x(k) \in \bar{U}\left[\inf P\left(K_{N-1}, k\right), \sup P\left(K_{N-1}, k\right)\right] \subset \bar{U}\left[\underline{x}^{N-1}, \bar{x}^{N-1}\right]=\left[\underline{g}\left(\bar{x}^{N-1}\right), \bar{g}\left(\underline{x}^{N-1}\right)\right]=\left[\underline{x}^{N}, \bar{x}^{N}\right]$,

where the inclusion follows from the monotonicity of $\bar{U}$, and the first equality follows from lemma 1. Statement (2) follows immediately, because $\underline{x}=\lim _{N} \underline{x}^{N}$ and $\bar{x}=\lim _{N} \bar{x}^{N}$.

Lemmas 1 through 3 help formalize one of the main results in this paper.

Theorem 1. In GSS, the following are equivalent.

1. Best response dynamic starting at inf $X($ or $\sup X)$ converges

2. Every adaptive dynamic converges to the same Nash equilibrium

3. Every adaptive dynamic converges to a Nash equilibrium

Moreover, in each case, the game has a unique Nash equilibrium.

Proof. We need only check that (1) implies (2). Let $\left(y^{k}\right)$ be the best response dynamic starting at $\inf X$. Then $\left(y^{2 k}\right)$ is a subsequence of the convergent sequence $\left(\underline{x}^{k}\right)$ and $\left(y^{2 k+1}\right)$ is a subsequence of the convergent sequence $\left(\bar{x}^{k}\right)$, and therefore, if $\left(y^{k}\right)$ converges, then $\underline{x}=$ $\bar{x}$. Moreover, $\underline{x} \in g(\bar{x})$, because if $\underline{x} \notin g(\bar{x})$, then there is $i$, and $x_{i}$ such that $f^{i}\left(x_{i}, \bar{x}_{-i}\right)-$ $f^{i}\left(\underline{x}_{i}, \bar{x}_{-i}\right)>0$. But then, by upper semi-continuity in the $i$ variable, and continuity in the $-i$ variables, for all $k$ sufficiently large, $f^{i}\left(x_{i}, \bar{x}_{-i}^{k}\right)-f^{i}\left(\underline{x}_{i}^{k+1}, \bar{x}_{-i}^{k}\right)>0$, contradicting the optimality of $\underline{x}_{i}^{k+1}$. Consequently, $\underline{x}$ is a Nash equilibrium. The previous lemma now implies that every adaptive dynamic converges to this Nash equilibrium. The proof is similar for the best response dynamic starting at $\sup X$.

If every adaptive dynamic converges to the same Nash equilibrium, the game has a unique Nash equilibrium. For if there were two distinct Nash equilibria, then consider the following two constant sequences; each playing one of the Nash equilibria. These are two adaptive dynamics converging to distinct Nash equilibria, a contradiction.

Theorem 1 provides a new perspective on global stability. Say that a strategic game $\Gamma$ is globally stable, if it has a (pure strategy) Nash equilibrium such that every adaptive dynamic converges to this Nash equilibrium. ${ }^{17}$ Intuitively, in a globally stable game, all adaptive learning dynamics and strategic processes always lead to the same outcome. Theorem 1 says that in GSS, convergence of the best response dynamic from inf (or sup) of the strategy space is equivalent to global stability. ${ }^{18}$

Theorem 1 shows that in GSS, global stability can be analyzed using a single best response dynamic, as an alternative to the traditional eigen-value approach; confer, for example, AlNowaihi and Levine (1985) and Okuguchi and Yamazaki (2008). Recall that eigen-value

\footnotetext{
${ }^{17}$ As shown in the proof of theorem 1, if a game is globally stable, then it has a unique Nash equilibrium.

${ }^{18}$ In GSC, theorem 1 is not necessarily true. Indeed, in GSC, part (1) of theorem 1 is always true.
} 
analysis requires making assumptions about each dynamic being analyzed. Theorem 1, however, yields convergence of all adaptive dynamics from knowledge of convergence of a single best response dynamic. Consequently, whether players actually play best response dynamics or not, convergence of a single best response dynamic is sufficient to conclude convergence under all adaptive behavior.

Theorem 1 helps provide a connection to existing results on dominance solvability in GSS, as follows. Recall that Zimper (2007) has shown that when each player $i$ 's payoff function is supermodular in $x_{i}$ and has decreasing differences in $\left(x_{i} ; x_{-i}\right)$, and when for each player $i$, there exists an order-continuous best response function, a GSS always has extremal serially undominated strategies, and a GSS is dominance solvable iff the second iterate of the (joint) best response function has a unique fixed point. The model we use here is more general. Payoff functions here are more general: quasi-supermodular in $x_{i}$ and satisfy the decreasing single crossing property in $\left(x_{i} ; x_{-i}\right){ }^{19}$ Moreover, we make assumptions on the primitive payoff functions, not on best responses. Furthermore, we do not make the implicit assumptions of convex strategy spaces and strictly quasi-concave payoffs to guarantee uniqueness of best responses; we work with best response correspondences.

In the next result, we show that Zimper's results (existence of extremal serially undominated strategies and equivalences 1-3 in theorem 2 below) go through for our generalizations. The proof is given in the appendix.

Theorem 2. Let $\Gamma$ be a GSS, $\underline{x}$ and $\bar{x}$ be the extremal serially undominated strategies, and $g$ be the joint best-response correspondence. The following are equivalent.

\section{1. $\Gamma$ is dominance solvable}

2. $\underline{x}=\bar{x}$

3. $g \circ g$ has a unique fixed point

4. Best response dynamic starting at inf $X($ or $\sup X)$ converges

Moreover, in each case, the game has a unique Nash equilibrium.

Combining theorems 1 and 2 yields the following connection.

Corollary 1. In GSS, the following are equivalent.

1. Best response dynamic starting at inf $X($ or $\sup X)$ converges

2. The game is globally stable

3. The game is dominance solvable

\footnotetext{
${ }^{19}$ The decreasing single crossing property is an ordinal generalization of decreasing differences.
} 
In particular, in GSS, both dominance solvability and global stability can be checked using a single best response dynamic.

Corollary 1 brings together two different foundations for robustness of predicted outcomes in games, in terms of convergence of a single best response dynamic. Dominance solvability (and rationalizability) assumes fully informed players, using infinite iterations of rationalizing about potential (future) responses by competitors to predict a solution to a one-shot game. Global stability uses dynamic learning and strategic processes in a series of game-play over time, using past play by typically myopic players to determine present moves, and relying on limits of such learning and strategic behavior to predict an outcome robust to the dynamics. In GSS, both approaches are equivalent, and moreover, these equivalences are accessible from knowledge of convergence of a single best response dynamic. ${ }^{20}$

Recall that Moulin (1984) has shown that in all strategic games, dominance solvability implies Cournot stability (convergence of all best response dynamics). Zimper (2007)'s results imply that in GSS, Cournot stability is equivalent to dominance solvability. Our results extend this to show that in GSS, global stability (convergence of every adaptive dynamic, not just best response dynamics) is equivalent to dominance solvability.

Fixed points of $g \circ g$ play a significant role in the analysis of GSS. These may be motivated in terms of strategy profiles that are rationalizable with short cycles of justification, in the spirit of Bernheim (1984). That is, suppose $x \in g \circ g(x)$. In this case, let $y \in g(x)$ such that $x \in g(y)$. Then the profile of strategies $x$ is rationalizable with the following cycle of conjectures. For each $i$, player $i$ plays $x_{i}$ because she believes her opponents shall play $y_{-i}$, because each of her opponents $j$ further believes that his opponents shall play $x_{-j}$. Say that a profile of strategies $x$ is simply rationalizable, if there is a strategy profile $y$ such that for every player $i, x_{i}$ can be justified by such a short cycle of conjectures.

The reasoning above shows that if a profile of strategies $x$ is a fixed point of $g \circ g$, then it is simply rationalizable. In the other direction, it is easy to check that if for each $i$, player $i$ plays $x_{i}$ because that is a best response to her belief that her opponents shall play $y_{-i}$, because each of her opponents $j$ best responds with $y_{j}$ based on his further belief that his opponents shall play $x_{-j}$, then the profile of strategies $x$ is a (joint) best response to the profile of strategies $y$, and $y$ is a best response to $x$, whence $x$ is a fixed point of $g \circ g$.

Thus, we may view fixed points of $g \circ g$ as strategy profiles that can be (simultaneously) rationalized by no more than two iterations of behavioral conjectures.

Intuitively, simply rationalizable strategies do not rely on high orders of deduction. They may be viewed as outcomes of low-level rationalization, exhibiting a type of bounded rationality. Nash equilibria are simply rationalizable ( $x$ and $y$ are the same), but in general, simply

\footnotetext{
${ }^{20}$ Using Milgrom and Roberts (1990), it is easy to see that as stated, the equivalence of parts (2) and (3) of corollary 1 is true for GSC as well. What is not true for GSC is the equivalence of (1) and (2), and the equivalence of (1) and (3)). In particular, in GSC, the best response dynamic from inf (respectively, sup) of the strategy space always converges to the smallest (respectively, largest) Nash equilibrium, and therefore, in GSC, convergence of either (or even both) best response dynamic does not necessarily imply global stability or dominance solvability.
} 
rationalizable strategies may include more strategies than Nash strategies. ${ }^{21}$ Moreover, simply rationalizable strategies may form only a strict subset of all rationalizable strategies, because rationalizable strategies include conjectural cycles of all orders. ${ }^{22}$

In this terminology, in GSS, simple rationalization always predicts an outcome (even if a GSS has no Nash equilibrium). Moreover, if simple rationalization predicts a unique outcome, then higher-level rationalization has no additional benefit.

\section{Applications}

Combining the equivalences in theorem 1, theorem 2, and corollary 1 allow for powerful cross-derivation of results. For example, if it is easy to know that a GSS has a unique profile of simply rationalizable strategies, then we may conclude that the game is globally stable, and every adaptive dynamic converges to the unique equilibrium. Similarly, if we can compute the convergence of the best-response dynamic from the inf (or sup) of the strategy space, we may conclude that the game is globally stable, and it is dominance solvable. A direct computation of best-response dynamics may be useful in other cases. The following applications explore these ideas.

Example 2 (Simple Cournot oligopoly). Consider a 3-firm Cournot oligopoly with linear inverse demand, $p=a-b\left(x_{1}+x_{2}+x_{3}\right)$, constant marginal cost, $c>0$, and with production capacity constrained to $\left[0, x^{\max }\right]$ for each firm. For range of the best-responses to be in the strategy space, suppose $x^{\max }=\frac{a-c}{2 b}$. In this case, the joint best response function is given by $g\left(x_{1}, x_{2}, x_{3}\right)=\left(\frac{a-c-b\left(x_{2}+x_{3}\right)}{2 b}, \frac{a-c-b\left(x_{1}+x_{3}\right)}{2 b}, \frac{a-c-b\left(x_{1}+x_{2}\right)}{2 b}\right)$, and the unique Nash equilibrium is given by $\left(x_{1}, x_{2}, x_{3}\right)=\left(\frac{a-c}{4 b}, \frac{a-c}{4 b}, \frac{a-c}{4 b}\right)$. Moreover, $g \circ g\left(x_{1}, x_{2}, x_{3}\right)=$ $\left(\frac{2 x_{1}+x_{2}+x_{3}}{4}, \frac{x_{1}+2 x_{2}+x_{3}}{4}, \frac{x_{1}+x_{2}+2 x_{3}}{4}\right)$, and it is easy to see that every point on the diagonal of $\left[0, x^{\max }\right]^{3}$ is a fixed point of $g \circ g$. In particular, $(0,0,0)$ is the smallest simply rationalizable strategy and $\left(\frac{a-c}{2 b}, \frac{a-c}{2 b}, \frac{a-c}{2 b}\right)$ is the largest. Thus, this game is neither globally stable, nor Cournot stable, nor dominance solvable. ${ }^{23}$ In fact, the best-response dynamic starting at $(0,0,0)$ cycles with $\left(\frac{a-c}{2 b}, \frac{a-c}{2 b}, \frac{a-c}{2 b}\right)$, and serially undominated strategies provide no help in narrowing the range of predicted outcomes.

Notice that in this example, the (joint) best response function is linear (technically, it is affine, but deferring to standard terminology, we term it linear). As linear best responses arise in other contexts, too, the next example provides a general result for this case.

\footnotetext{
${ }^{21}$ Zimper (2007) provides an example of a three-player, three-action GSS with a unique Nash equilibrium, but with at least two simply rationalizable strategy profiles. A simple, textbook Cournot oligopoly example is provided in example 2 below.

${ }^{22}$ Indeed, in the original example in Bernheim (1984), there are rationalizable strategies that are not simply rationalizable. Our approach may be extended to define order- $n$ rationalizable strategies as fixed points of the $n$-th iterate of $g$. Order-1 strategies are Nash profiles, order- 2 strategies are simply rationalizable, and so on. We need only order-2 strategies in this paper, and mention their behavioral properties. Higher order rationalizability is not needed for this paper, and is not explored here.

${ }^{23}$ Recall that Zimper (2007) provides an example of a three-player, three-action GSS with a unique Nash equilibrium, and in which every strategy profile is serially undominated.
} 
Example 3 (Linear best responses). Consider a GSS with finitely many players, $N$, where each $X^{i}=\left[0, x_{i}^{\max }\right] \subset \mathbb{R}$, and $X=\left[0, x^{\max }\right] \subset \mathbb{R}^{N} \cdot{ }^{24}$ Suppose the joint best response function is affine; that is $g: X \rightarrow X$ such that $g(x)=a+B x$, where each component of the $N \times N$ matrix $B$ is non-positive. Thus, $g$ is nonincreasing. Moreover, for range of $g$ to be in $X$, suppose $-B x^{\max } \leq a \leq x^{\max }$. Notice that the game has a unique equilibrium, if, and only if, the matrix $(I-B)$ is invertible. In this case, the unique equilibrium is $x^{*}=(I-B)^{-1} a$. The iterated best response is given by $g \circ g(x)=a+B a+B^{2} x$, and therefore, $g \circ g$ has a unique fixed point, if, and only if, the matrix $\left(I-B^{2}\right)$ is invertible. As determinant calculations are easy to make, this result is useful in applications. Consider the following examples.

Example 3-1 (Cournot oligopoly; linear demand, linear cost). The 3-firm Cournot oligopoly in example 2 is a special case of example 3 , with $B=\left[\begin{array}{ccc}0 & -\frac{1}{2} & -\frac{1}{2} \\ -\frac{1}{2} & 0 & -\frac{1}{2} \\ -\frac{1}{2} & -\frac{1}{2} & 0\end{array}\right]$. It is easy to check that $I-B$ is invertible, but $I-B^{2}$ is not. Thus, the game has a unique Nash equilibrium, but is neither globally stable nor dominance solvable.

Example 3-2 (Cournot oligopoly; linear demand, quadratic cost). Consider a 3-firm Cournot oligopoly with linear inverse demand, as in example 2 , but with quadratic cost, $c x_{i}^{2}$, with $c>0$, and with production capacity constrained to $\left[0, x^{\max }\right]$ for each firm. In this case, the joint best-response function is given by $g\left(x_{1}, x_{2}, x_{3}\right)=\left(\frac{a-b\left(x_{2}+x_{3}\right)}{2 b+2 c}, \frac{a-b\left(x_{1}+x_{3}\right)}{2 b+2 c}, \frac{a-b\left(x_{1}+x_{2}\right)}{2 b+2 c}\right)$. Therefore, $B=\left[\begin{array}{ccc}0 & -\frac{b}{2 b+2 c} & -\frac{b}{2 b+2 c} \\ -\frac{b}{2 b+2 c} & 0 & -\frac{b}{2 b+2 c} \\ -\frac{b}{2 b+2 c} & -\frac{b}{2 b+2 c} & 0\end{array}\right]$. Let $\xi=\left(\frac{b}{2 b+2 c}\right)^{2}$. Then $\frac{b}{2 b+2 c}<\frac{1}{2}$ implies $\xi<\frac{1}{4}$. Therefore, $\operatorname{det}\left(I-B^{2}\right)=(1-2 \xi)\left(1-4 \xi+\xi^{2}\right)-2 \xi^{3}>(1-2 \xi) \xi^{2}-2 \xi^{3}=\xi^{2}(1-4 \xi)>0$. Consequently, this oligopoly is globally stable, the dominance solution (and several other solution concepts nested within the dominance solution) predicts a unique outcome, and the unique Nash equilibrium is robust to all adaptive behavior.

Example 3-3 (Common-pool resource game). Another game that shares the same structure is the common-pool resource game. ${ }^{25}$ Consider a 3-player common-pool resource game. Each player has an endowment $w>0$. There are two investment options - a common resource (such as a fishery) that exhibits diminishing marginal return, and an outside option with diminishing marginal return. If player $i$ invests an amount $x_{i} \leq w$ of his endowment into the common resource, he receives a proportional share of the total output $\frac{x_{i}}{x_{1}+x_{2}+x_{3}}\left(a\left(x_{1}+x_{2}+x_{3}\right)-b\left(x_{1}+x_{2}+x_{3}\right)^{2}\right)$, and he receives $r\left(w-x_{i}\right)-s\left(w-x_{i}\right)^{2}$ on the outside investment $w-x_{i}{ }^{26}$ Thus, payoff to player $i$ is

$f^{i}\left(x_{1}, x_{2}, x_{3}\right)=r\left(w-x_{i}\right)-s\left(w-x_{i}\right)^{2}+\frac{x_{i}}{x_{1}+x_{2}+x_{3}}\left(a\left(x_{1}+x_{2}+x_{3}\right)-b\left(x_{1}+x_{2}+x_{3}\right)^{2}\right)$,

if $x_{1}+x_{2}+x_{3}>0$, and $r w-s w^{2}$, otherwise. Notice that best response of player $i$ is given by $g^{i}\left(x_{j}, x_{k}\right)=\frac{a-r+2 s w}{2 b+2 s}-\frac{b}{2 b+2 s}\left(x_{j}+x_{k}\right)$. For range of $g^{i}$ to lie in $[0, w]$, we assume

\footnotetext{
${ }^{24}$ Here, $x^{\max }$ is the vector with $i$-th component $x_{i}^{\max }$.

${ }^{25}$ See, for example, Ostrom, Gardner, and Walker (1994). Additional analysis of this game as a GSS is presented in Roy and Sabarwal (2010).

${ }^{26}$ Here, $a, b, r, s>0$.
} 
$\frac{a-r}{2 b} \leq w \leq \frac{a-r}{2(b-s)} \cdot{ }^{27}$ Matrix $B$ is given by $B=\left[\begin{array}{ccc}0 & -\frac{b}{2 b+2 s} & -\frac{b}{2 b+2 s} \\ -\frac{b}{2 b+2 s} & 0 & -\frac{b}{2 b+2 s} \\ -\frac{b}{2 b+2 s} & -\frac{b}{2 b+2 s} & 0\end{array}\right]$. As in example $3-2, I-B^{2}$ is invertible, the game is globally stable, the unique equilibrium is robust to all adaptive behavior, and several solution concepts all predict the same unique equilibrium outcome.

Example 3-4 (Private provision of public goods). Suppose there are finitely many consumers, indexed $i=1, \ldots, N$, and there are two goods - good 1 is numeraire, indexed $y$, and good 2 is a public good, indexed $x$, and all variables are measured in terms of the numeraire (or in units of account). Suppose consumer $i$ 's utility is Cobb-Douglas, given by $u^{i}\left(y_{i}, x_{1}, \ldots, x_{N}\right)=y_{i}^{\alpha}\left(x_{1}+\ldots+x_{N}\right)^{\beta}$, where $\alpha, \beta>0$. Each consumer's budget constraint is $y_{i}+x_{i}=w_{i}$. Substituting $x_{i}-w_{i}$ for $y_{i}$, it is easy to calculate that player 1's best response function is given by $g^{1}\left(x_{2}, \ldots, x_{N}\right)=\frac{\beta w_{1}}{\alpha+\beta}-\frac{\alpha}{\alpha+\beta}\left(x_{2}+\ldots+x_{N}\right)$, and similarly for the other players. To ensure best-responses remain non-negative, we impose the constraint $\alpha(N-1) w^{\max } \leq \beta w_{i}$, for each player $i$. (Here, $w^{\max }=\max _{i} w_{i}$.) The matrix $B$ is given by $B=\frac{1}{\alpha+\beta}\left[\begin{array}{cccc}0 & -\alpha & \cdots & -\alpha \\ -\alpha & 0 & \cdots & -\alpha \\ \vdots & \vdots & \ddots & \vdots \\ -\alpha & -\alpha & \cdots & 0\end{array}\right]$. It is easy to check that each entry of $B^{2}$ is nonnegative, and moreover, the sum of each row of $B^{2}$ is $\left(\frac{(N-1) \alpha}{\alpha+\beta}\right)^{2}$, which is strictly less than one. Therefore, $\sum_{n=0}^{\infty}\left(B^{2}\right)^{n}=\left(I-B^{2}\right)^{-1}$. Consequently, this game is globally stable, and dominance solvable. A similar example can be worked out with utility given by constant elasticity of substitution.

Example 3-5 (Differentiated goods Cournot oligopoly). Consider finitely many firms, indexed $i=1, \ldots, N$, each facing demand curve $p_{i}=\alpha-\beta x_{i}-\delta\left(x_{1}+\ldots+x_{i-1}+x_{i+1}+\ldots x_{N}\right)$, each with constant marginal cost $c$, and production constrained to $\left[0, x^{\max }\right]$. To introduce differentiated goods, assume that $\beta \neq \delta$; that is, the impact of self production on price is different from the impact of competitor output on price. It is easy to calculate that firm 1's best response function is given by $g^{1}\left(x_{2}, \ldots, x_{N}\right)=\frac{\alpha-c}{2 \beta}-\frac{\delta}{2 \beta}\left(x_{2}+\ldots+x_{N}\right)$, and similarly for the other players. To ensure best-responses remain in the strategy space, we assume that $\frac{\alpha-c}{2 \beta}<x^{\max }$ and that $\alpha-c \geq(N-1) \delta x^{\max }$. The matrix $B$ is given by $B=\frac{1}{2 \beta}\left[\begin{array}{cccc}0 & -\delta & \cdots & -\delta \\ -\delta & 0 & \cdots & -\delta \\ \vdots & \vdots & \ddots & \vdots \\ -\delta & -\delta & \cdots & 0\end{array}\right]$. As in the previous example, it is easy to check that each entry of $B^{2}$ is non-negative, and moreover, the sum of each row of $B^{2}$ is $\left(\frac{(N-1) \delta}{2 \beta}\right)^{2}$, which is strictly less than one. Therefore, $\sum_{n=0}^{\infty}\left(B^{2}\right)^{n}=\left(I-B^{2}\right)^{-1}$, and this game is globally stable, and dominance solvable, and every adaptive dynamic converges to the unique Nash equilibrium. Notice that additional heterogeneity can be introduced in this example by varying $\alpha, \beta, \delta, x^{\max }$ and $c$ by firm. Moreover, a similar example can be formulated using quadratic, or more general costs.

\footnotetext{
${ }^{27}$ Hence, we need $a>r$ and $b>s$.
} 
Applications to games with non-linear best-responses are considered in the following examples.

Example 4 (Tournaments). Suppose a tournament ${ }^{28}$ has 3 players, where a reward $r>0$ is shared by the players who succeed in the tournament. If one player succeeds, he gets $r$ for sure, if two players succeed, each gets $r$ with probability one-half, and if all players succeed, each gets $r$ with probability one-third. Each player chooses effort $x_{i} \in[0,1]$ with probability of success $x_{i}$. Expected reward per unit for player $i$ is

$$
\pi^{i}\left(x_{i}, x_{j}, x_{k}\right)=x_{i}\left(1-x_{j}\right)\left(1-x_{k}\right)+\frac{1}{2} x_{i} x_{j}\left(1-x_{k}\right)+\frac{1}{2} x_{i} x_{k}\left(1-x_{j}\right)+\frac{1}{3} x_{i} x_{j} x_{k} .
$$

The quadratic cost of effort $x_{i}$ is $c x_{i}^{2}$. The payoff to player $i$ is expected reward minus cost of effort. That is, $f^{i}\left(x_{i}, x_{j}, x_{k}\right)=r \pi^{i}\left(x_{i}, x_{j}, x_{k}\right)-c x_{i}^{2}$. It is easy to calculate that best response of player $i$ is given by $g^{i}\left(x_{j}, x_{k}\right)=\frac{r}{2 c}\left(1-\frac{1}{2}\left(x_{j}+x_{k}\right)+\frac{1}{3} x_{j} x_{k}\right)$. Suppose, for convenience, $r=2 c$.

This game is globally stable, as follows. Notice that if a best response dynamic starts anywhere on the diagonal in $[0,1]^{3}$, then it remains entirely on the diagonal. This reduces the problem to checking convergence only on the diagonal. Using, $x_{1}=x_{2}=x_{3}=x$, say, this reduces the problem to checking if the iterated dynamic given by $\gamma(x)=1-x+\frac{1}{3} x^{2}$ converges. (Here, $\gamma$ is the best-response of an arbitrary player, reduced to one-dimension, using symmetry.) Notice that $\left|\gamma^{\prime}(x)\right|=1-\frac{2}{3} x<1$, if $x>0$. Therefore, $\gamma$ satisfies the contraction principle over $[\epsilon, 1+\epsilon]$ for every sufficiently small $\epsilon>0$. In particular, the best-response dynamic starting at $(1,1,1)$ converges. Consequently, every adaptive dynamic converges, the game is globally stable, and dominance solvable. (For reference, the unique globally stable equilibrium is $(3-\sqrt{6}, 3-\sqrt{6}, 3-\sqrt{6})$.)

Example 5 (General symmetric Cournot oligopoly). Consider a $N$-firm Cournot oligopoly, with inverse demand curve given by $P\left(x_{1}+\ldots+x_{N}\right)$, cost of firm $i$ given by $C\left(x_{i}\right)$, both these functions are twice continuously differentiable, and production is constrained to $\left[0, x^{\max }\right]$. Assume that demand is downward sloping and cost is convex. The first-order condition for firm $i$ is given by $x_{i} P^{\prime}+P-C^{\prime}=0$, and therefore, the slope of the best response of firm $i$ with respect to firm $j$ 's output is given by $-\frac{x_{i} P^{\prime \prime}+P^{\prime}}{x_{i} P^{\prime \prime}+2 P^{\prime}-C^{\prime \prime}}$. This is a GSS, if $x_{i} P^{\prime \prime}+P^{\prime}<0$.

As in the tournaments example above, if a best response dynamic starts anywhere on the diagonal in $\left[0, x^{\max }\right]^{N}$, then it remains entirely on the diagonal. This reduces the problem to checking convergence only on the diagonal. Write the best-response dynamic implicitly as $y P^{\prime}(y+(N-1) x)+P(y+(N-1) x)-C^{\prime}(y)=0$, where $y$ is the best response to each competitor playing $x$. This process is globally asymptotically stable, if $\left|\frac{d y}{d x}\right|<1$, which is satisfied if $\left|C^{\prime \prime}-P^{\prime}\right|>(N-2)\left|y P^{\prime \prime}+P^{\prime}\right|$. In this case, this game is dominance solvable and the unique equilibrium is globally stable under all adaptive behavior. This result is valid for large $N$, as compared to, for example, Al-Nowaihi and Levine (1985).

Let's apply this result to a specific functional form. As in Amir (1996), suppose inverse demand is given by $P\left(x_{1}+\ldots+x_{N}\right)=\frac{1}{\left(x_{1}+\ldots+x_{N}+1\right)^{\alpha}}$, where $\alpha>0$, and cost is given by

\footnotetext{
${ }^{28}$ This version is based on Dubey, Haimanko, and Zapechelnyuk (2006). Additional analysis of this game as a GSS is presented in Roy and Sabarwal (2010).
} 
$C\left(x_{i}\right)=\frac{1}{2} c x_{i}^{2}$. In this case, best responses are downward sloping, if $0<\alpha<\frac{1}{x^{\max }}$. Moreover, the condition $\left|C^{\prime \prime}-P^{\prime}\right|>(N-2)\left|y P^{\prime \prime}+P^{\prime}\right|$ is automatically satisfied for $N=2$, 3. More generally, it is satisfied, if $c>(N-3) \alpha$. Thus, the best response dynamic on the diagonal converges, if $\alpha<\min \left\{\frac{c}{N-3}, \frac{1}{x^{\max }}\right\}$. In particular, if we normalize production so that $x^{\max }=1$, then the condition holds for $0<\alpha<1$, and $c>N-3$. Notice that this result is valid for large $N$, as compared to, for example, Al-Nowaihi and Levine (1985).

Example 6 (Two-player GSS). For two-player GSS, global stability is equivalent to uniqueness of Nash equilibrium, as follows. It is known that in a two-player GSS, dominance solvability is equivalent to uniqueness of Nash equilibrium, because a two-player GSS can be viewed as a GSC by reversing the order on the strategy space of one of the players. Corollary 1 then yields the desired equivalence. ${ }^{29}$

Example 7 (Two-player GSC). Notice that a two-player GSC may be viewed as a GSS by reversing the order on the strategy space of one of the players. Therefore, the results here apply to such games. In particular, (in the original order in a two-player GSC,) if the best-response dynamic starting from $\left(\inf X_{1}, \sup X_{2}\right)\left(\right.$ or from $\left(\sup X_{1}, \inf X_{2}\right)$ ) converges, then the game is globally stable, and dominance solvable. Similarly, if a two-player GSC has a unique Nash equilibrium, it is globally stable.

In some games (especially discrete games), it may be easy to carry out a direct computation of best response dynamics, as shown in the next example.

Example 8 (Dove-Hawk-Chicken-2). Consider the Dove-Hawk-Chicken game (example 1 ), but with slightly modified payoffs given in figure 2 . The only modification is that in the top row of each matrix, player 2's payoffs are flipped. This results in $D$ as the dominant action for player 2. Intuitively, player 2 is a type that prefers less conflict (or avoids aggression, or would prefer a more "cooperative" action).

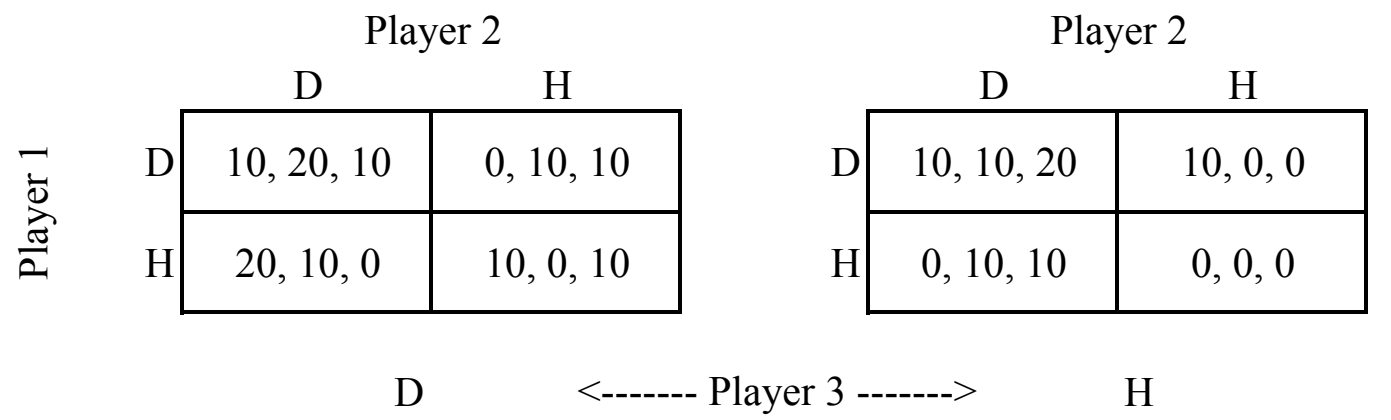

Figure 2: Dove-Hawk-Chicken-2

It is easy to check that $(D, D, H)$ is the unique equilibrium in this game. Moreover, the best response dynamic starting from $(D, D, D)$ converges after two iterations: $(D, D, D) \mapsto$

\footnotetext{
${ }^{29}$ As shown above, this result does not extend to games with more than two players; Zimper (2007) provides a three-player, three-action counter-example, and the 3-firm Cournot oligopoly with linear demand and constant marginal cost (example 2) provides a counter-example. Similarly, although a Nash equilibrium is guaranteed in a 2-player GSS, existence of equilibrium does not extend to more than two players, as shown in the Dove-Hawk-Chicken game (example 1).
} 
$(H, D, H) \mapsto(D, D, H)$. Consequently, this game is dominance solvable, and the unique equilibrium is globally stable under all adaptive behavior.

The connections shown in this paper provide several equivalent techniques to check for robustness of equilibrium predictions in GSS. Uniqueness of simply rationalizable strategies may be easy to check in games with linear best responses, convergence of best response dynamics may be easy to check in cases with non-linear best responses, especially in the presence of some symmetry, and a direct computation of best response dynamics may be useful in discrete games.

Notice that specialized results are possible in particular situations. For example, as shown in Jensen (2009), in strictly quasi-concave aggregative games with strategic substitutes, with one-dimensional strategy sets, and with a unique pure strategy Nash equilibrium, sequential best-reply dynamics converge to the unique Nash equilibrium. ${ }^{30}$ As sequential best reply dynamics are adaptive dynamics, this result shows that in particular cases, a subset of the set of adaptive dynamics may be well-behaved. Such a result is useful, if we have in mind particular subclasses of dynamics and wish to determine if these are well-behaved.

\section{Stability of Monotone Equilibrium Selections}

To formalize ideas of dynamic stability of equilibria in parameterized games with strategic substitutes, we need some notions about best response dynamics starting at arbitrary points in the strategy space.

Let $\Gamma$ be a GSS, and $y \preceq z$ be elements of $X$. The (simultaneous) best response dynamic-1 starting at $y$ is the sequence $\left(y^{k}\right)_{k=0}^{\infty}$, where $y^{0}=y$, and for $k \geq 1, y^{k}=\underline{g}\left(y^{k-1}\right)$ if $n$ is even, and $y^{k}=\bar{g}\left(y^{k-1}\right)$ if $n$ is odd. Similarly, the (simultaneous) best response dynamic- 2 starting at $z$ is the sequence $\left(z^{k}\right)_{k=0}^{\infty}$, where $z^{0}=z$, and for $k \geq 1, z^{k}=\bar{g}\left(z^{k-1}\right)$ if $n$ is even, and $z^{k}=\underline{g}\left(z^{k-1}\right)$ if $n$ is odd. Notice that when $y=\inf X$, best response dynamic- 1 is the best response dynamic starting at inf $X$, and when $z=\sup X$, best response dynamic-2 is the best response dynamic starting at $\sup X$. Moreover, both best response dynamics coincide when $g$ is a function and $y=z$.

Given $y \preceq z$ and best response dynamics 1 and $2,\left(y^{k}\right)$ and $\left(z^{k}\right)$, the definition of the lower and upper mixtures of $\left(\left(y^{k}\right) ;\left(z^{k}\right)\right)$ remains the same; $\left(\underline{x}^{k}\right)_{k=0}^{\infty}$ is given by $\underline{x}^{k}=y^{k}$, if $k$ is even, and $\underline{x}^{k}=z^{k}$, if $k$ is odd, and $\left(\bar{x}^{k}\right)_{k=0}^{\infty}$ is given by $\bar{x}^{k}=z^{k}$, if $k$ is even, and $\bar{x}^{k}=y^{k}$, if $k$ is odd.

The definition of an adaptive dynamic is similar. Following Echenique (2002), a process $(x(k))_{k=0}^{\infty}$ is an adaptive dynamic in the game $\Gamma$ if there is $\gamma>0$ such that for all $k \geq 0$, $x(k) \in \bar{U}[\inf P(k-\gamma, k)$, sup $P(k-\gamma, k)]$, where as earlier, $P(k-\gamma, k)$ is the history of past play from $k-\gamma$ to $k$; that is, $P(k-\gamma, k)=\{x(k-\gamma), x(k-\gamma+1), \ldots, x(k-1)\}$. By convention, when $\gamma \geq k$, we set $k-\gamma=0$. It is easy to check that this is a special case of our earlier definition, using discrete time and a uniform bound on the length of history to

\footnotetext{
${ }^{30}$ Jensen (2009)'s result does not extend to simultaneous best-reply dynamics, as shown by the Cournot oligopoly with linear demand and linear cost (example 2) above.
} 
affect a current decision. ${ }^{31}$

The following lemma presents a generalization of lemma 3 . Its proof is given in the appendix. The proof builds on techniques from Echenique (2002), adjusted for the special challenges that arise when dealing with strategic substitutes.

Lemma 4. Let $y \preceq z,\left(y^{k}\right)$ and $\left(z^{k}\right)$ be best response dynamics 1 and 2 , respectively, and $\left(\underline{x}^{k}\right)$ and $\left(\bar{x}^{k}\right)$ be their lower and upper mixtures, respectively. For every $x^{0} \in[y, z]$, and for every adaptive dynamic $(x(k))$ starting at $x^{0}$, the following is true.

1. For every $N$, there is $K_{N}$, such that for all $k \geq K_{N}, x(k) \in\left[\underline{x}^{N}, \bar{x}^{N}\right]$.

2. If $y^{0} \preceq y^{2}$, then there exist simply rationalizable $\underline{y}, \bar{y}$ such that

$$
\underline{y} \preceq \liminf x(k) \preceq \limsup x(k) \preceq \bar{y} .
$$

3. If $z^{2} \preceq z^{0}$, then there exist simply rationalizable $\underline{z}, \bar{z}$ such that

$$
\underline{z} \preceq \liminf x(k) \preceq \lim \sup x(k) \preceq \bar{z}
$$

Notice the initial monotonicity condition in parts 2 and 3 . These conditions are automatically satisfied when $y^{0}=\inf X$ and $z^{0}=\sup X$, as was the case earlier, but may not necessarily be satisfied more generally. Below, we shall consider and motivate cases when these initial monotonicity conditions are satisfied.

Parameterized games with strategic substitutes are defined as follows. As earlier, consider a set of players $I$, and for each player $i$, a partially ordered strategy space $\left(X^{i}, \preceq^{i}\right)$, and the overall strategy space $X$.

Moreover, consider a partially ordered set of parameters, $T .{ }^{32}$ We restrict the parameter space to satisfy a basic "density" property; that is, we assume that for every order interval $[\underline{t}, \bar{t}]$ in $T$ and for every $\hat{t}$ such that $\underline{t} \prec \hat{t} \prec \bar{t}$, every neighborhood of $\hat{t}$ contains $t_{0}, t_{1} \in[\underline{t}, \bar{t}]$ such that $t_{0} \prec \hat{t} \prec t_{1}$. Notice that this property is fairly basic. In particular, a convex $T \subset \mathbb{R}^{n}$, as assumed in Echenique (2002), is admissible. This property rules out parameter spaces where order intervals contain isolated points.

Each player $i$ has a payoff function, $f^{i}: X \times T \rightarrow \mathbb{R}$, denoted $f^{i}\left(x_{i}, x_{-i}, t\right)$. The collection $\Gamma=\left(I, T,\left(X^{i}, \preceq^{i}, f^{i}\right)_{i \in I}\right)$ is a parameterized game with strategic substitutes, (parameterized $\boldsymbol{G S S}$ ), if for every player $i$,

- $\left(X^{i}, \preceq^{i}\right)$ is a complete lattice,

- For every $\left(x_{-i}, t\right), f^{i}$ is order upper semi-continuous in $x_{i}$, and for every $x_{i}, f^{i}$ is order continuous in $\left(x_{-i}, t\right)$,

\footnotetext{
${ }^{31}$ This specialization is helpful in generalizing lemma 3. See the double-induction argument in the proof of lemma 4.

${ }^{32}$ For convenience, the partial order on $T$ is denoted by the same symbol, $\preceq$, and $T$ is assumed to have the standard order interval topology.
} 
- For every $\left(x_{-i}, t\right), f^{i}$ is quasi-supermodular in $x_{i}$,

- For every $x_{-i}, f^{i}$ satisfies single-crossing property in $\left(x_{i} ; t\right)$, and

- For every $t, f^{i}$ satisfies decreasing single-crossing property in $\left(x_{i} ; x_{-i}\right)$.

As usual, single-crossing property in $\left(x_{i} ; t\right)$ implies that each player's best response, $g^{i}\left(x_{-i}, t\right)$ is nondecreasing in the parameter, a standard formulation. As earlier, each player's best response is nonincreasing in other player strategies. Thus, the joint best response, $g(x, t)$ is nondecreasing in $t$ and nonincreasing in $x$.

As usual, for each $t \in T$, a parameterized GSS, $\Gamma$, naturally defines a GSS, $\Gamma(t)$, with the same strategy spaces as $\Gamma$ and with appropriate sections of the payoff functions. Let $\mathcal{E}(t)$ denote the set of (pure strategy) Nash equilibria in $\Gamma(t)$.

An equilibrium selection is a function $e: T \rightarrow X$ such that for every $t, e(t) \in \mathcal{E}(t)$. An equilibrium selection $e: T \rightarrow X$ is nowhere weakly increasing on $[\underline{t}, \bar{t}]$, if for every $t_{0}, t_{1} \in[\underline{t}, \bar{t}], t_{0} \prec t_{1}$ implies $e\left(t_{0}\right) \npreceq e\left(t_{1}\right) .{ }^{33}$ An equilibrium selection $e: T \rightarrow X$ is strictly increasing if it is nondecreasing and for every $t_{0} \prec \hat{t} \prec t_{1},\left[e\left(t_{0}\right), e\left(t_{1}\right)\right]$ is a neighborhood of $e(\hat{t})$ in $X .{ }^{34}$ For notational convenience, we sometimes denote $g(\cdot, t)$ as $g_{t}(\cdot)$.

As is well-known, parameterized GSS do not necessarily exhibit monotone comparative statics, and therefore, nondecreasing equilibrium selections do not necessarily exist in such games. Roy and Sabarwal (2010) provide intuitive conditions that guarantee monotone comparative statics in parameterized GSS, as follows.

Let $\Gamma$ be a parameterized GSS. An equilibrium selection $e: T \rightarrow X$ satisfies condition 1 on $[\underline{t}, \bar{t}]$, if for every $t_{0}, \hat{t}$ in $[\underline{t}, \bar{t}]$ such that $t_{0} \preceq \hat{t}, e\left(t_{0}\right) \preceq \underline{g}\left(\bar{g}\left(e\left(t_{0}\right), \hat{t}\right), \hat{t}\right)$. An equilibrium selection $e: T \rightarrow X$ satisfies condition 2 on $[\underline{t}, \bar{t}]$, if for every $\hat{t}, t_{1}$ in $[\underline{t}, \bar{t}]$ such that $\hat{t} \preceq t_{1}$, $\bar{g}\left(\underline{g}\left(e\left(t_{1}\right), \hat{t}\right), \hat{t}\right) \preceq e\left(t_{1}\right)$.

As shown in Roy and Sabarwal (2010), in GSS, conditions 1 and 2 present a natural tradeoff between a direct parameter effect and an indirect strategic substitute effect, as follows. Suppose $g$ is a function. Starting from an existing equilibrium, $e\left(t_{0}\right)$ at $t=t_{0}$, an increase in $t$ to $\hat{t}$ has two effects on, say, player $i$ 's best response function, $g^{i}(\cdot, \cdot)$. The direct parameter effect is an increase in $g^{i}$, because best-response is nondecreasing in $t$. The indirect strategic substitute effect is a decrease in $g^{i}$, because an increase in $t$ increases the best response of the competitors of $i$, and their actions are strict substitutes for player $i$. Thus, $e\left(t_{0}\right) \preceq g\left(g\left(e\left(t_{0}\right), \hat{t}\right), \hat{t}\right)$ in condition 1 says that for each player, the indirect strategic substitute effect does not dominate the direct parameter effect when the parameter goes up. Condition 2 makes the analogous statement when the parameter goes down. ${ }^{35}$

\footnotetext{
${ }^{33}$ As described in Echenique (2002), this is stronger than the negation of weakly increasing.

${ }^{34}$ When $X$ is in some finite dimensional Euclidean space, as in Echenique (2002), this definition is equivalent to $t_{0} \prec t_{1} \Rightarrow e\left(t_{0}\right) \ll e\left(t_{1}\right)$. Another relevant case is when $X$ is a subset of a Banach lattice that has a positive cone with a nonempty interior.

${ }^{35}$ For GSS, Roy and Sabarwal (2010) present conditions on payoff functions and on best responses under which the above conditions hold. Notice that in GSC, both effects work in the same direction. Therefore, once the direct parameter effect is assumed to be favorable, (as formalized, for example, by a strict single
} 
Conditions 1 and 2 are useful to apply lemma 4 , as follows. Consider $t_{0} \preceq \hat{t}$. If condition 1 is satisfied, then the best response dynamic- 1 given by $y^{0}=e\left(t_{0}\right)$, and for $k \geq 1, y^{k}=$ $\underline{g}\left(y^{k-1}, \hat{t}\right)$ if $n$ is even, and $y^{k}=\bar{g}\left(y^{k-1}, \hat{t}\right)$ if $n$ is odd has the feature that $y^{0} \preceq y^{2}$. Similarly, consider $\hat{t} \preceq t_{1}$. If condition 2 is satisfied then the best response dynamic-2 given by $z^{0}=$ $e\left(t_{1}\right)$, and for $k \geq 1, z^{k}=g\left(z^{k-1}, \hat{t}\right)$ if $n$ is odd, and $z^{k}=\bar{g}\left(z^{k-1}, \hat{t}\right)$ if $n$ is even has the feature that $z^{2} \preceq z^{0}$. This allows us to use lemma 4 .

To state and prove an analogue of the correspondence principle for GSS, consider the following notions of stability. Let $\Gamma$ be a parameterized GSS and $t \in T$. A point $\hat{x} \in X$ is weakly stable at $t$, if there is a neighborhood $V$ of $\hat{x}$ such that for every $x \in V$, there is an adaptive dynamic $(x(k))$ in $\Gamma(t)$ that starts at $x$ and converges to $\hat{x}$. A point $\hat{x} \in X$ is strongly stable at $t$, if there is a neighborhood $V$ of $\hat{x}$ such that for every $x \in V$, every adaptive dynamic $(x(k))$ in $\Gamma(t)$ that starts at $x$ converges to $\hat{x}$. For notational convenience, we sometimes denote $g(\cdot, t)$ as $g_{t}(\cdot)$.

Theorem 3. (Correspondence Principle) Let $\Gamma$ be a parameterized GSS and e be a continuous equilibrium selection.

(1) If e is nowhere weakly increasing and satisfies condition 1 on $[\underline{t}, \bar{t}]$, then for every $\hat{t}$ such that $\underline{t} \prec \hat{t} \prec \bar{t}, e(\hat{t})$ is not weakly stable at $\hat{t}$.

(2) If e is strictly increasing and satisfies conditions 1 and 2 on $[\underline{t}, \bar{t}]$, then for every $\hat{t}$ such that $\underline{t} \prec \hat{t} \prec \bar{t}$ and $e(\hat{t})$ is an isolated fixed point of $g_{\hat{t}} \circ g_{\hat{t}}$, e $(\hat{t})$ is strongly stable at $\hat{t}$.

Proof. Consider (1). Fix $\hat{t}$ such that $\underline{t} \prec \hat{t} \prec \bar{t}$. Consider $e(\hat{t})$, and an arbitrary neighborhood $V$ of $e(\hat{t})$. By continuity of $e$, let $t_{0}$ be such that $\underline{t} \preceq t_{0} \prec \hat{t}$ and $e\left(t_{0}\right) \in V$. Then, by nowhere weakly increasing, $e\left(t_{0}\right) \npreceq e(\hat{t})$. Consider an arbitrary adaptive dynamic $(x(k))$ in $\Gamma(\hat{t})$ starting at $x(0)=e\left(t_{0}\right)$. Let $y^{0}=e\left(t_{0}\right)$ and for $k \geq 1, y^{k}=\underline{g}_{\hat{t}}\left(y^{k-1}\right)$ if $n$ is even, and $y^{k}=\bar{g}_{\hat{t}}\left(y^{k-1}\right)$ if $n$ is odd. By condition $1, y^{0} \preceq y^{2}$. Therefore, by lemma 4 , $e\left(t_{0}\right) \preceq \underline{y} \preceq \liminf x(k)$, whence $x(k) \nrightarrow e(\hat{t})$.

Consider (2). Fix $\hat{t}$ such that $\underline{t} \prec \hat{t} \prec \bar{t}$ and $e(\hat{t})$ is an isolated fixed point of $g_{\hat{t}} \circ g_{\hat{t}}$. Let $N$ be a neighborhood of $e(\hat{t})$ such that $N \cap \mathcal{E}(\hat{t})=\{e(\hat{t})\}$. As $e$ is continuous, let $t_{0}, t_{1} \in[\underline{t}, \bar{t}]$ be such that $t_{0} \prec \hat{t} \prec t_{1}$, and $e\left(t_{0}\right)$ and $e\left(t_{1}\right)$ are in $N$. As $e$ is strictly increasing, $\left[e\left(t_{0}\right), e\left(t_{1}\right)\right]$ is a neighborhood of $e(\hat{t})$. Consequently, $V=\left[e\left(t_{0}\right), e\left(t_{1}\right)\right] \cap N$ is a neighborhood of $e(\hat{t})$ and $e(\hat{t})$ is the only fixed point of $g_{\hat{t}} \circ g_{\hat{t}}$ in $V$.

Fix $x^{0} \in V$ arbitrarily, and let $(x(k))$ be an arbitrary adaptive dynamic in $\Gamma(\hat{t})$ starting at $x^{0}$. Let $\left(y^{k}\right)$ and $\left(z^{k}\right)$ be best response dynamics 1 and 2 , respectively, with $y^{0}=e\left(t_{0}\right)$ and $z^{0}=e\left(t_{1}\right)$. Using conditions 1 and 2 , and lemma 4 , it follows that

$$
e\left(t_{0}\right)=y^{0} \preceq \underline{y} \preceq \liminf x(k) \preceq \lim \sup x(k) \preceq \bar{z} \preceq z^{0}=e\left(t_{1}\right),
$$

whence $\underline{y}$ and $\bar{z}$ are in $\left[e\left(t_{0}\right), e\left(t_{1}\right)\right]$. As $\underline{y}$ and $\bar{z}$ are fixed points of $g_{\hat{t}} \circ g_{\hat{t}}$, by local isolation, $\underline{y}=\bar{z}=\bar{e}(\hat{t})$. Thus, $x(k) \rightarrow e(\hat{t})$, as desired.

Theorem 3 provides conditions under which strict monotone comparative statics select equilibria that are dynamically stable, in the sense that for small changes in the parameter, at

crossing property in $\left(x_{i} ; t\right)$,) the indirect strategic complement effect serves to reinforce the direct effect, and the conditions above are satisfied. 
a new parameter value, every adaptive dynamic starting from the old equilibrium converges to the newly selected equilibrium. Moreover, nowhere increasing selections select equilibria that are dynamically unstable, in the sense that at a new parameter value, no adaptive dynamic starting from the old equilibrium converges to the newly selected equilibrium. Thus, when considering dynamically stable equilibria (as proposed by Samuelson's Correspondence principle), we may expect monotone selections of equilibria to arise naturally in GSS.

Notice that Echenique (2002) shows these results for parameterized GSC without using conditions 1 and 2, but implicitly assuming a strict single-crossing property (correspondences are assumed to be strongly increasing in $t$ ). The results here show the importance of focusing on an appropriate tradeoff between the direct parameter effect and the indirect strategic effect rather than on a strict single-crossing property. As described above, for parameterized GSC, a strict single-crossing property leads to a strong direct parameter effect, and the indirect effect does not matter anymore, because it serves to reinforce the direct effect. For parameterized GSS, it is precisely the reversed nature of the indirect strategic substitute effect that requires conditions 1 and 2 to be useful in proving the analogous results. Indeed, in this case, we do not require a strict single-crossing property and correspondences are not assumed to be strongly increasing in $t$.

Example 9 (Team projects with substitutable tasks). Suppose a project is to be accomplished by a team of 3 players, ${ }^{36}$ each choosing task (or effort) $x_{i} \in[0,1]$, with probability of success $x_{i}$ and quadratic cost of effort $\frac{c}{2} x_{i}^{2}$, with $c>0$. Tasks are substitutable in the sense that each player by herself can make the project successful. The probability of success is $1-\left(1-x_{1}\right)\left(1-x_{2}\right)\left(1-x_{3}\right)$. If the project is successful, player $i$ receives a parameterized reward $r(t)>0$ (with $t \in T$, a compact, convex order interval in $\mathbb{R}$, and $\left.r^{\prime}(t)>0.\right)^{37}$ Otherwise, the player receives zero. Therefore, the payoff to player $i$ is $f^{i}\left(x_{1}, x_{2}, x_{3}, t\right)=r(t)\left(1-\left(1-x_{1}\right)\left(1-x_{2}\right)\left(1-x_{3}\right)\right)-\frac{c}{2} x_{i}^{2}$.

The best response of player $i$ is $g^{i}\left(x_{j}, x_{k}, t\right)=\frac{r(t)}{c}\left(1-x_{j}\right)\left(1-x_{k}\right)$. For notational convenience, let $a(t)=\frac{r(t)}{c}$, and when convenient, we suppress the notation $t$. To ensure that best responses remain in the strategy space, we assume that $a \leq 1$ (for every $t$ ). In fact, we assume that $a<\frac{3}{4}$, the reason becoming clear below.

For each $t$, this game has a unique symmetric equilibrium, which is strictly increasing in $t$, as follows. Fix $t$. First, observe that in any equilibrium, no player plays 0 or 1 , as follows. Suppose, $x_{1}=1$ in equilibrium. Then using player 2's and 3's best response function, $x_{2}=x_{3}=0$, whence, $x_{1}=a<1$, a contradiction. Similarly, $x_{2} \neq 1$ and $x_{3} \neq 1$. Suppose $x_{1}=0$ in equilibrium. Then using player 1's best response function, either $x_{2}=1$ or $x_{3}=1$, but that contradicts $x_{2} \neq 1$ and $x_{3} \neq 1$. Second, observe that only symmetric equilibria (each player plays the same action) are possible, as follows. Suppose, in equilibrium, $x_{1}>x_{2}$. Then using player 1's best response, $x_{1}+a x_{2}-a x_{2} x_{3}=a-a x_{3}$, and using player 2's best response, $x_{2}+a x_{1}-a x_{1} x_{3}=a-a x_{3}$, whence $(1-a)\left(x_{1}-x_{2}\right)=a\left(x_{2} x_{3}-x_{1} x_{3}\right)$, a contradiction. Thus

\footnotetext{
${ }^{36}$ This version is based on Dubey, Haimanko, and Zapechelnyuk (2006).

${ }^{37}$ The parameter $t$ can be viewed as technological improvement, or subsidy provided, or reward provided to induce an increase in effort (or probability) of task completion. As shown in the example, the best response function depends on $\frac{r(t)}{c_{i}}$, where $c_{i}$ measures player $i$ 's costs, and therefore, $r(t)$ can be viewed as a reward enhancement parameter relative to a player's costs.
} 
$x_{1}=x_{2}$. Similarly, $x_{2}=x_{3}$. Third, observe that a symmetric equilibrium exists, and is given by $\frac{2 a+1-\sqrt{4 a+1}}{2 a}$ for each player. Fourth, as shown by Roy and Sabarwal (2008) symmetric equilibria in GSS are unique, so this game has a unique equilibrium. Finally, it is easy to check that this equilibrium selection is strictly increasing in $t$.

Consequently, this equilibrium selection is strictly increasing and selects locally isolated equilibria.

Let's check that conditions 1 and 2 are satisfied. Fix $t_{0}$ in the interior of $T$, and let $x^{*}$ denote the equilibrium selection at $t_{0}$. Notice that for $t \geq t_{0}, \gamma\left(x^{*}, t\right)=a(t)\left(1-x^{*}\right)^{2}$, where $\gamma$ is the best-response of a player, reduced to one-dimension, using symmetry. Therefore, $\gamma\left(\gamma\left(x^{*}, t\right), t\right)=a(t)\left[1-\frac{a(t)}{a\left(t_{0}\right)} a\left(t_{0}\right)\left(1-x^{*}\right)^{2}\right]^{2}=a(t)\left[1-\frac{a(t)}{a\left(t_{0}\right)} x^{*}\right]^{2}$, where the last equality follows from $x^{*}=a\left(t_{0}\right)\left(1-x^{*}\right)^{2}$. Using $a^{\prime}>0$ and $x^{*}<1$, it is easy to check that $\left.\frac{d}{d t} \gamma\left(\gamma\left(x^{*}, t\right), t\right)\right|_{t=t_{0}}>0$, if, and only if, $x^{*}<\frac{1}{3}$. Using $x^{*}=\frac{2 a+1-\sqrt{4 a+1}}{2 a}$, this condition is satisfied when $a<\frac{3}{4}$, as assumed above. Consequently, for every $t_{0}$ in the interior of $T$, there is a neighborhood $[\underline{t}, \bar{t}] \subset T$ of $t_{0}$ such that conditions 1 and 2 are satisfied on $[\underline{t}, \bar{t}]$. Applying the theorem above, for every $t_{0}$ in the interior of $T, e\left(t_{0}\right)$ is strongly stable.

A similar example can be constructed using tournaments as well. 


\section{References}

Acemoglu, D., And M. K. Jensen (2009): "Aggregate comparative statics," Working Paper, Department of Economics, University of Birmingham and MIT.

(2010): "Robust comparative statics in large static games," Working Paper, Department of Economics, University of Birmingham.

Al-Nowaini, A., And P. Levine (1985): "The Stability of the Cournot Oligopoly Model: A Reassessment," Journal of Economic Theory, 35, 307-321.

Aliprantis, C. D., And K. C. Border (1994): Infinite Dimensional Analysis: A Hitchhiker's Guide. Springer-Verlag.

Amir, R. (1996): "Cournot Oligopoly and the Theory of Supermodular Games," Games and Economic Behavior, 15, 132-148.

Amir, R., And V. E. Lambson (2000): "On the Effects of Entry in Cournot Markets," The Review of Economic Studies, 67(2), 235-254.

Bernheim, B. D. (1984): "Rationalizable Strategic Behavior," Econometrica, 52(4), 10071028.

Bulow, J. I., J. D. Geanakoplos, and P. D. Klemperer (1985): "Multimarket Oligopoly: Strategic Substitutes and Complements," Journal of Political Economy, 93(3), $488-511$.

Dubey, P., O. Haimanko, and A. Zapechelnyuk (2006): "Strategic complements and substitutes, and potential games," Games and Economic Behavior, 54, 77-94.

EChenique, F. (2002): "Comparative statics by adaptive dynamics and the correspondence principle," Econometrica, 70(2), 257-289.

Echenique, F., And T. Sabarwal (2003): "Strong Comparative Statics of Equilibria," Games and Economic Behavior, 42(2), 307-314.

Edlin, A., And C. Shannon (1998): "Strict Monotonicity in Comparative Statics," Journal of Economic Theory, 81(1), 201-219.

Jensen, M. K. (2009): "Stability of pure strategy Nash equilibrium in best-reply potential games," Working Paper, Department of Economics, University of Birmingham.

$45-66$.

Lippman, S. A., J. W. Mamer, and K. F. MCCardle (1987): "Comparative Statics in non-cooperative games via transfinitely iterated play," Journal of Economic Theory, 41(2), 288-303. 
Milgrom, P., And J. Roberts (1990): "Rationalizability, learning, and equilibrium in games with strategic complementarities," Econometrica, 58(6), 1255-1277.

— (1994): "Comparing Equilibria," American Economic Review, 84(3), 441-459.

Milgrom, P., and C. Shannon (1994): "Monotone Comparative Statics," Econometrica, 62(1), 157-180.

Moulin, H. (1984): "Dominance Solvability and Cournot Stability," Mathematical Social Sciences, 7(1), 83-102.

OkUguchi, K., and T. Yamazaki (2008): "Global stability of unique Nash equilibrium in Cournot oligopoly and rent-seeking game," Journal of Economic Dynamics and Control, $32,1204-1211$.

Ostrom, E., R. Gardner, and J. Walker (1994): Rules, Games, and Common-Pool Resources. The University of Michigan Press, Ann Arbor.

QuAh, J. K.-H. (2007): "The Comparative Statics of Constrained Optimization Problems," Econometrica, 75(2), 401-431.

Quah, J. K.-H., and B. Strulovici (2009): "Comparative statics, informativeness, and the interval dominance order," Econometrica, 77(6), 1949-1992.

Roy, S., And T. Sabarwal (2008): "On the (Non-)Lattice Structure of the Equilibrium Set in Games With Strategic Substitutes," Economic Theory, 37(1), 161-169.

_ (2010): "Monotone comparative statics for games with strategic substitutes," Journal of Mathematical Economics, forthcoming.

Schipper, B. C. (2003): "Submodularity and the evolution of Walrasian behavior," International Journal of Game Theory, 32, 471-477.

Shannon, C. (1995): "Weak and Strong Monotone Comparative Statics," Economic Theory, 5(2), 209-227.

Sobel, J. (1988): "Isotone comparative statics in supermodular games," Mimeo. SUNY at Stony Brook.

TOPKIS, D. (1978): "Minimizing a submodular function on a lattice," Operations Research, $26,305-321$.

(1979): "Equilibrium points in nonzero-sum n-person submodular games," SIAM Journal on Control and Optimization, 17(6), 773-787.

(1998): Supermodularity and Complementarity. Princeton University Press.

Villas-Boas, J. M. (1997): "Comparative Statics of Fixed Points," Journal of Economic Theory, 73(1), 183-198. 
VIVEs, X. (1990): "Nash Equilibrium with Strategic Complementarities," Journal of Mathematical Economics, 19(3), 305-321.

— (1999): Oligopoly Pricing. MIT Press.

(2005): "Complementarities and Games: New Developments," Journal of Economic Literature, 43(2), 437-479.

Zhou, L. (1994): "The Set of Nash Equilibria of a Supermodular Game is a Complete Lattice," Games and Economic Behavior, 7(2), 295-300.

ZIMPER, A. (2007): "A fixed point characterization of the dominance-solvability of lattice games with strategic substitutes," International Journal of Game Theory, 36(1), 107-117. 


\section{Appendix}

Lemma. Let $\Gamma$ be a GSS. Let $\left(\left(y^{k}\right) ;\left(z^{k}\right)\right)$ be the best response dynamics starting at inf $X$ and $\sup X$, respectively, and let $\underline{x}$ and $\bar{x}$ be the limits of the lower and upper mixtures of $\left(\left(y^{k}\right) ;\left(z^{k}\right)\right)$, respectively. Then $\underline{x}$ and $\bar{x}$ are the smallest and largest profiles of serially undominated strategies, respectively.

Proof. Let $U^{0}(X)=X$, and for $k \geq 1$, let $U^{k}(X)=U\left(U^{k-1}(X)\right)$, where $U(S)$ is the collection of undominated responses to $S$. It follows by induction that for $k \geq 0, U^{k}(X) \subset\left[\underline{x}^{k}, \bar{x}^{k}\right]$, as follows. This holds trivially for $k=0$. Suppose it holds for $k-1$. Then for $k$,

$$
U^{k}(X)=U\left(U^{k-1}(X)\right) \subset U\left[\underline{x}^{k-1}, \bar{x}^{k-1}\right] \subset\left[\underline{g}\left(\bar{x}^{k-1}\right), \bar{g}\left(\underline{x}^{k-1}\right)\right]=\left[\underline{x}^{k}, \bar{x}^{k}\right],
$$

where the first inclusion follows from the inductive hypothesis and monotonicity of $U$, and the second inclusion follows from lemma 1. Consequently, $\bigcap_{k=0}^{\infty} U^{k}(X) \subset[\underline{x}, \bar{x}]$. That is, the set of serially undominated strategies is contained in the order interval $[\underline{x}, \bar{x}]$.

Notice now that $\underline{x}$ is a best response to $\bar{x}$, and $\bar{x}$ is a best response to $\underline{x}$. That is, $\underline{x} \in g(\bar{x})$ and $\bar{x} \in g(\underline{x})$, as follows. Suppose $\underline{x} \notin g(\bar{x})$. Then there is $i$, and $x_{i}$ such that $f^{i}\left(x_{i}, \bar{x}_{-i}\right)-f^{i}\left(\underline{x}_{i}, \bar{x}_{-i}\right)>0$. But then, by upper semi-continuity in the $i$ variable, and continuity in the $-i$ variables, for all $k$ sufficiently large, $f^{i}\left(x_{i}, \bar{x}_{-i}^{k}\right)-f^{i}\left(\underline{x}_{i}^{k+1}, \bar{x}_{-i}^{k}\right)>0$, contradicting the optimality of $\underline{x}_{i}^{k+1}$. Similarly, $\bar{x} \in g(\underline{x})$.

Finally, note that $\underline{x}$ and $\bar{x}$ are in $\bigcap_{k=0}^{\infty} U^{k}(X)$, as follows. Trivially, $\underline{x}$ and $\bar{x}$ are in $U^{0}(X)$. Suppose $\underline{x}$ and $\bar{x}$ are in $U^{k}(X)$. Then $\underline{x} \in U^{k+1}(X)$, because $\bar{x} \in U^{k}(X)$ and $\underline{x}$ is a best response to $\bar{x}$, and $\bar{x} \in U^{k+1}(X)$, because $\underline{x} \in U^{k}(X)$ and $\bar{x}$ is a best response to $\underline{x}$. Thus, serially undominated strategies lie in $[\underline{x}, \bar{x}]$, and the end points are extremal serially undominated strategies.

Theorem 2. Let $\Gamma$ be a GSS, $\underline{x}$ and $\bar{x}$ be the extremal serially undominated strategies, and $g$ be the joint best-response correspondence. The following are equivalent.

\section{1. $\Gamma$ is dominance solvable}

2. $\underline{x}=\bar{x}$

3. $g \circ g$ has a unique fixed point

4. Best response dynamic from $\inf X($ or $\sup X)$ converges

Moreover, in each case, the game has a unique Nash equilibrium.

Proof. Let $F P(g \circ g)$ denote the set of fixed point of $g \circ g$, and notice that $F P(g \circ g) \subset \bigcap_{k=0}^{\infty} U^{k}(X)$, as follows. Consider an arbitrary $x \in g \circ g(x)$. Let $y \in g(x)$ be such that $x \in g(y)$. Then, by induction, $x$ and $y$ are in $\bigcap_{k=0}^{\infty} U^{k}(X)$, as follows. Trivially, $x$ and $y$ are in $U^{0}(X)$. Suppose $x$ and $y$ are in $U^{k}(X)$. Then $x \in U^{k+1}(X)$, because $y \in U^{k}(X)$ and $x$ is a best response to $y$, and $y \in U^{k+1}(X)$, because $x \in U^{k}(X)$ and $y$ is a best response to $x$. Consequently, we have the following relationships: $\mathcal{E} \subset F P(g \circ g) \subset \bigcap_{k=0}^{\infty} U^{k}(X) \subset[\underline{x}, \bar{x}]$. 
Notice next that both $\underline{x}$ and $\bar{x}$ are fixed points of $g \circ g$, because $\underline{x} \in g(\bar{x})$ and $\bar{x} \in g(\underline{x})$. With this observation, (3) implies (2) implies (1) follows immediately. For (1) implies (3), notice that if the game is dominance solvable, then the serially undominated strategies $\underline{x}$ and $\bar{x}$ are the same, and the chain of inclusions above shows that $g \circ g$ has a unique fixed point. The equivalence of (2) and (4) is shown in the text; confer lemma 3, and the proof of theorem 1.

Lemma 4. Let $y \preceq z,\left(y^{k}\right)$ and $\left(z^{k}\right)$ be best response dynamics 1 and 2 , respectively, and $\left(\underline{x}^{k}\right)$ and $\left(\bar{x}^{k}\right)$ be their lower and upper mixtures, respectively. For every $x^{0} \in[y, z]$, and for every adaptive dynamic $(x(k))$ starting at $x^{0}$, the following is true.

1. For every $N$, there is $K_{N}$, such that for all $k \geq K_{N}, x(k) \in\left[\underline{x}^{N}, \bar{x}^{N}\right]$.

2. If $y^{0} \preceq y^{2}$, then there exist simply rationalizable $\underline{y}, \bar{y}$ such that

$$
\underline{y} \preceq \liminf x(k) \preceq \lim \sup x(k) \preceq \bar{y} .
$$

3. If $z^{2} \preceq z^{0}$, then there exist simply rationalizable $\underline{z}, \bar{z}$ such that

$$
\underline{z} \preceq \liminf x(k) \preceq \lim \sup x(k) \preceq \bar{z}
$$

Proof. To prove statement (1), consider $N=0$. Let $K_{0}=0$. Notice that $x(0)=x^{0} \in\left[y^{0}, z^{0}\right]$, by assumption, and $\left[y^{0}, z^{0}\right]=\left[\underline{x}^{0}, \bar{x}^{0}\right]$, by construction. Suppose for $0 \leq k \leq \hat{k}-1, x(k) \in\left[\underline{x}^{0}, \bar{x}^{0}\right]$. Then $P(0, \hat{k}) \subset\left[\underline{x}^{0}, \bar{x}^{0}\right]$, whence

$$
\begin{aligned}
x(\hat{k}) & \in \bar{U}[\inf P(\hat{k}-\gamma, \hat{k}), \sup P(\hat{k}-\gamma, \hat{k})] \\
& \subset \bar{U}[\inf P(0, \hat{k}), \sup P(0, \hat{k})] \\
& \subset \bar{U}\left[\underline{x}^{0}, \bar{x}^{0}\right] \subset\left[\underline{x}^{0}, \bar{x}^{0}\right],
\end{aligned}
$$

where membership follows from definition of an adaptive dynamic, the first inclusion follows from $P(\hat{k}-\gamma, \hat{k}) \subset P(0, \hat{k})$ and monotonicity of $\bar{U}$, the second inclusion follows from the inductive hypothesis and monotonicity of $\bar{U}$, and the last inclusion follows trivially. Thus, for all $k \geq 0$, $x(k) \in\left[\underline{x}^{0}, \bar{x}^{0}\right]$.

Suppose the statement is true for $N-1$. Let $K_{N-1}$ be given by the inductive hypothesis. Let $K_{N}=K_{N-1}+\gamma$, where $\gamma$ is from the definition of adaptive dynamic. Suppose $N$ is even. Fix $\hat{k} \geq K_{N}=K_{N-1}+\gamma$. Then

$$
\begin{aligned}
x(\hat{k}) & \in \bar{U}[\inf P(\hat{k}-\gamma, \hat{k}), \sup P(\hat{k}-\gamma, \hat{k})] \\
& \subset \bar{U}\left[\inf P\left(K_{N-1}, \hat{k}\right), \sup P\left(K_{N-1}, \hat{k}\right)\right] \\
& \subset \bar{U}\left[\underline{x}^{N-1}, \bar{x}^{N-1}\right]=\left[\underline{x}^{N}, \bar{x}^{N}\right],
\end{aligned}
$$

where membership follows from definition of an adaptive dynamic, the first inclusion follows from $P(\hat{k}-\gamma, \hat{k}) \subset P\left(K_{N-1}, \hat{k}\right)$ and monotonicity of $\bar{U}$, the second inclusion follows from the inductive hypothesis and monotonicity of $\bar{U}$, and the equality follows from lemma 1 . Thus, for all $k \geq K_{N}$, $x(k) \in\left[\underline{x}^{N}, \bar{x}^{N}\right]$.

To prove statement (2), notice first that $y^{0} \preceq y^{2}$ implies that the subsequence $\left(y^{2 k}\right)$ is nondecreasing, and by completeness, there is $y$ such that $y^{2 k} \rightarrow y$. Similarly, using $y^{0} \preceq y^{2} \Rightarrow y^{3}=$ $\bar{g}\left(y^{2}\right) \preceq \bar{g}\left(y^{0}\right)=y^{1}$, the subsequence $\left(y^{2 k-1}\right)$ is nonincreasing, and there is $\bar{y}$ such that $\lim _{k} y^{2 k}=\bar{y}$.

Notice next that $\underline{y}, \bar{y} \in F P(g \circ g)$. This follows from the observation that $\underline{y} \in g(\bar{y})$, and $\bar{y} \in g(\underline{y})$, as follows. Suppose $\underline{y} \notin g(\bar{y})$. Then there is $i$, and $x_{i}$ such that $f^{i}\left(x_{i}, \bar{y}_{-i}\right)-f^{i}\left(\underline{y}_{i}, \bar{y}_{-i}\right)>0$. But 
then, by upper semi-continuity in the $i$ variable, and continuity in the $-i$ variables, for all $k$ sufficiently large, $f^{i}\left(x_{i}, y_{-i}^{2 k-1}\right)-f^{i}\left(y_{i}^{2 k}, y_{-i}^{2 k-1}\right)>0$, contradicting the optimality of $y_{i}^{2 k}$. Similarly, $\bar{y} \in g(\underline{y})$. Consequently, $\underline{y}, \bar{y}$ are simply rationalizable.

Consider an arbitrary $x^{0} \in[y, z]$, and an arbitrary adaptive dynamic $(x(k))$ starting at $x^{0}$. Consider an arbitrary convergent subsequence $\left(x\left(k_{l}\right)\right)$ of $(x(k))$. By the lemma 4 , for $N=0$, there is $K_{0}$ such that for all $k_{l} \geq K_{0}, y^{0} \preceq x\left(k_{l}\right)$, whence $y^{0}=\underline{x}^{0} \preceq \lim _{l} x\left(k_{l}\right)$. For $N=2$, there is $K_{2}$ such that for all $k_{l} \geq K_{2}, y^{2}=\underline{x}^{2} \preceq x\left(k_{l}\right)$, whence $y^{2} \preceq \lim _{l} x\left(k_{l}\right)$. And by induction, for $2 N$, there is $K_{2 N}$ such that for all $k_{l} \geq K_{2 N}, y^{2 N}=\underline{x}^{2 N} \preceq x\left(k_{l}\right)$, whence $y^{2 N} \preceq \lim _{l} x\left(k_{l}\right)$. Consequently, $\underline{y} \preceq \lim _{l} x\left(k_{l}\right)$. As $\left(x\left(k_{l}\right)\right)$ is an arbitrary convergent subsequence, it follows that $\underline{y} \preceq \lim \inf x(k)$. Moreover, as $\underline{x}$ is the smallest fixed point of $g \circ g$, it follows that $\underline{x} \preceq \underline{y}$. Similarly, $\overline{\lim \sup } x(k) \preceq \bar{y} \preceq \bar{x}$. Statement (3) follows similarly. 